\title{
Computation of the Maximum Likelihood estimator in low rank Factor Analysis
}

\author{
Koulik Khamaru * $\quad$ Rahul Mazumder ${ }^{\dagger}$
}

January 19, 2018

\begin{abstract}
Factor analysis, a classical multivariate statistical technique is popularly used as a fundamental tool for dimensionality reduction in statistics, econometrics and data science. Estimation is often carried out via the Maximum Likelihood (ML) principle, which seeks to maximize the likelihood under the assumption that the positive definite covariance matrix can be decomposed as the sum of a low rank positive semidefinite matrix and a diagonal matrix with nonnegative entries. This leads to a challenging rank constrained nonconvex optimization problem. We reformulate the low rank ML Factor Analysis problem as a nonlinear nonsmooth semidefinite optimization problem, study various structural properties of this reformulation and propose fast and scalable algorithms based on difference of convex (DC) optimization. Our approach has computational guarantees, gracefully scales to large problems, is applicable to situations where the sample covariance matrix is rank deficient and adapts to variants of the ML problem with additional constraints on the problem parameters. Our numerical experiments demonstrate the significant usefulness of our approach over existing state-of-the-art approaches.
\end{abstract}

\section{Introduction}

Factor Analysis (FA) 2, 6, 24, arguably a fundamental dimensionality reduction technique for multivariate data has been around for more than a hundred years. FA is popularly used to understand the correlation structure among a collection of observed variables in terms of a smaller number of common factors. In a typical FA model, we assume that the (mean centered) observed random vector $\mathbf{x} \in \Re^{p \times 1}$ may be expressed in the form: $\mathbf{x}=\mathbf{L f}+\mathbf{u}$, where, $\mathbf{L}:=$ $\left(\left(\ell_{i j}\right)\right) \in \Re^{p \times r}$ is a matrix of factor loadings, $\mathbf{f} \in \Re^{r \times 1}$ is a random vector of scores and $\mathbf{u} \in \Re^{p \times 1}$ is a vector of uncorrelated variables. We assume that $\mathbf{f}$ and $\mathbf{u}$ are uncorrelated, have zero means and without loss of generality we set $\operatorname{Cov}(\mathbf{f})=\mathrm{I}_{\mathrm{r}}$-this leads to the following decomposition:

$$
\operatorname{Cov}(\mathbf{x}):=\mathbf{\Sigma}=\mathbf{L} \mathbf{L}^{\top}+\mathbf{\Psi}
$$

where, $\operatorname{Cov}(\mathbf{u}):=\boldsymbol{\Psi}=\operatorname{diag}\left(\psi_{1}, \ldots, \psi_{p}\right)$. Decomposition (1) suggests that the population covariance matrix $\boldsymbol{\Sigma}:=\left(\left(\sigma_{i j}\right)\right)$, can be written as the sum of a low rank positive semidefinite (PSD) matrix and a diagonal matrix $\boldsymbol{\Psi}$ with nonnegative entries. In particular, this implies that $\operatorname{Var}\left(x_{i}\right)$ can be decomposed in two parts $\operatorname{Var}\left(x_{i}\right)=\sigma_{i i}^{2}=\sum_{k=1}^{r} \ell_{i k}^{2}+\psi_{i}$, where, $\sum_{k=1}^{r} \ell_{i k}^{2}$

\footnotetext{
* Department of Statistics, University of California Berkeley. koulik@berkeley.edu

${ }^{\dagger}$ MIT Sloan School of Management, Operations Research Center and Center for Statistics; Massachusetts Institute of Technology. rahulmaz@mit .edu
} 
represents the variance of $x_{i}$ which is shared with other variables via common factors (communality) and $\psi_{i}$ represents the variance of $x_{i}$ not shared with other variables (specific or unique variance).

One of the most popular FA estimation methods is the Maximum Likelihood (ML) procedure [24, 2, 6]. Given $n$ multivariate samples $\mathbf{x}_{i}, i=1, \ldots, n$, which are assumed to be mean-centered, the task is to minimize the negative log-likelihood with respect to $\boldsymbol{\Sigma}$ that is of the form (1). This leads to the following optimization problem:

$$
\begin{aligned}
\operatorname{minimize} & \mathcal{L}(\boldsymbol{\Sigma}):=-\log \operatorname{det}\left(\boldsymbol{\Sigma}^{-1}\right)+\operatorname{tr}\left(\boldsymbol{\Sigma}^{-1} \mathbf{S}\right) \\
\text { s.t. } & \boldsymbol{\Sigma}=\mathbf{\Psi}+\mathbf{L} \mathbf{L}^{\top} \\
& \boldsymbol{\Psi}=\operatorname{diag}\left(\psi_{1}, \ldots, \psi_{p}\right) \succeq \epsilon \mathbf{I},
\end{aligned}
$$

where, $\mathbf{S}=\frac{1}{n} \sum_{i=1}^{n} \mathbf{x}_{i} \mathbf{x}_{i}^{\top}$ is the sample covariance matrix; $\boldsymbol{\Psi} \in \Re^{p \times p}, \mathbf{L} \in \Re^{p \times r}$ and $\boldsymbol{\Sigma}$ are the optimization variables; the notation $\mathbf{A} \succeq \mathbf{B}$ means that $\mathbf{A}-\mathbf{B}$ is PSD; and $\operatorname{tr}(\cdot)$ is the usual trace operator. Here, $\epsilon$ is a small positive constant, specified a-priori to ensure that Problem (2) is bounded below 1 . We note that Problem (2) can be rewritten in terms of a new variable $\boldsymbol{\Theta}=\mathbf{L L}^{\prime} \in \Re^{p \times p}$ and placing a rank constraint on $\boldsymbol{\Theta}$ as: $\operatorname{rank}(\boldsymbol{\Theta}) \leq r$ - hence, in what follows, we will often refer to Problem (2) as a rank constrained optimization problem.

Observe that Problem (2) is nonconvex in $\boldsymbol{\Sigma}$ since (a) the objective function $\mathcal{L}(\boldsymbol{\Sigma})$ is not convex [10] in $\boldsymbol{\Sigma}$ and (b) the equality constraint $\boldsymbol{\Sigma}=\boldsymbol{\Psi}+\mathbf{L} \mathbf{L}^{\top}$ is nonconvex.

Related work: FA has a long tradition that dates back to more than a hundred years 33. We present here a selective overview that is relevant for this work-important contributions in FA have been nicely documented in [24, 2, 6, 4. Despite being a problem of fundamental importance in statistical estimation, not much is known about its computational properties. Unfortunately, popular off-the-shelf implementations for ML factor analysis (as available in Matlab and $R$ that are in routine use) are quite unstable ${ }^{2}$ - they are based on rather ad-hoc computational algorithms and lead to negative variance estimates which are problematic from a statistical inferential viewpoint. This is perhaps not surprising, given that the basic problem underlying ML factor analysis is a difficult (nonconvex) optimization problem and there has been limited work in developing mathematical optimization based algorithms for this problem. It is also difficult to generalize existing algorithms in the presence of additional constraints, depending upon the problem/application context. 21, 2 present a nice overview of classical algorithms used for ML factor analysis. Some modern computational approaches for ML factor analysis are based on the seminal contribution of [17. This approach requires $\mathbf{S}$ to be of full rank and one needs to assume that $\mathbf{L}$ has exactly rank $r$. It applies a (rather ad-hoc) gradient descent like algorithm w.r.t $\boldsymbol{\Psi}$. Recently [28] provide necessary and sufficient conditions for existence of a solution of Problem (2) with $\epsilon=0$, however, they do not provide any computational algorithm for solving Problem (2). Another popular approach for ML factor analysis is based on the EM algorithm [30, 8]. Publicly available implementations of the EM-type methods apply to the case where, $n$ is smaller than $p$ and hence $\mathbf{S}$ is not full rank.

Not all methods in FA are based on the ML framework. In other approaches, one seeks to estimate a matrix $\boldsymbol{\Sigma}$ of the form $\boldsymbol{\Sigma}=\boldsymbol{\Psi}+\mathbf{L} \mathbf{L}^{\top}$, which is close to the sample covariance matrix $\mathbf{S}$ in terms of some metric (for e.g., the Frobenius norm: $\|\mathbf{S}-\boldsymbol{\Sigma}\|_{F}$ ). Some popular methods in the literature are minimum residual FA, principal axis, principal component method, minimum trace FA, among others - see [32, 6, 7] for more description on these approaches. Fairly recently [7]

\footnotetext{
${ }^{1}$ Indeed, $\boldsymbol{\Psi} \succeq \epsilon \mathbf{I}$ implies that $\boldsymbol{\Sigma} \succeq \epsilon \mathbf{I} \succ \mathbf{0}$. Thus, $-\log \operatorname{det}\left(\boldsymbol{\Sigma}^{-1}\right) \geq p \log (\epsilon)$ and $\operatorname{tr}\left(\boldsymbol{\Sigma}^{-1} \mathbf{S}\right) \geq 0$ which shows that Problem 2 is bounded below. Note that Problem 2 with $\epsilon=0$ need not have a finite solution, i.e., the ML solution need not exist. Note that if one of the $\psi_{i} \rightarrow+\infty$ then $\mathcal{L}(\boldsymbol{\Sigma}) \rightarrow \infty$, a similar argument applies if $\mathbf{L} \mathbf{L}^{\prime}$ becomes unbounded. Thus the infimum of Problem (2) is attained whenever $\epsilon>0$.

${ }^{2}$ We have observed this in our experiments and this is also reported in our section on numerical experiments.
} 
proposed integer optimization based methods for the problem of minimizing $\|\mathbf{S}-\boldsymbol{\Sigma}\|_{F}^{2}$, where, $\boldsymbol{\Sigma}$ is of the form (1) with an additional restriction of $\mathbf{S}-\boldsymbol{\Sigma} \succeq \mathbf{0}$. 31] study the noiseless decomposition for the FA problem, using nuclear norm relaxations of the rank constraint on $\Theta:=\mathbf{L L}^{\top}$. The aforementioned line of work is different from the ML criterion in FA as the data-fidelity measure is different. In this paper, our focus is on the computational properties of the ML problem (2).

Contributions: The main contributions of this paper can be summarized as follows:

1. We propose a new computational framework for the task of (Gaussian) Maximum Likelihood estimation in Factor Analysis - a problem central to classical and modern multivariate statistical learning. The associated optimization problem is challenging: it is given by the minimization of a nonconvex function subject to a rank constraint and additional semidefinite constraints. We reformulate the problem as the minimization of a nonsmooth nonconvex function of eigenvalues of a positive semidefinite matrix, subject to (simple) polyhedral constraints.

2. Using (convexity) properties of spectral functions, we show that the objective function can be expressed as a difference of convex functions; and is hence amenable to computational techniques in difference of convex optimization (see for example [16]). The computational bottleneck of our algorithm is a low rank singular value decomposition (SVD) of a $p \times p$ matrix that needs to be performed for every iteration - exploiting problem structure, we show that this can be computed with cost $O\left(\min \{n, p\}^{2} \max \{n, p\}\right)$. An important advantage of our proposal, when compared to many other commonly used, publicly available implementations; is that it applies to the case where the sample covariance matrix is rank deficient.

3. We explore computational guarantees of our proposed algorithm in terms of reaching a first order stationary point. We demonstrate that on a series of numerical examples (including both real and synthetic datasets), our method significantly outperforms commonly used approaches for ML factor analysis (in terms of superior numerical stability, smaller computation times and obtaining solutions with better objective values). To our knowledge, our proposal is one of the most scalable computational mathematical optimization-based approaches for factor analysis. Our approach also generalizes to instances of ML factor analysis where, $\boldsymbol{\Psi}$ is not necessarily diagonal.

Notation: For a real symmetric matrix $\mathbf{A}_{p \times p}$, we will denote by $\boldsymbol{\lambda}(\mathbf{A})$, the vector of real eigenvalues of $\mathbf{A}$, i.e., $\left(\lambda_{1}(\mathbf{A}), \lambda_{2}(\mathbf{A}), \ldots, \lambda_{p}(\mathbf{A})\right)$ with $\lambda_{i}(\mathbf{A}) \geq \lambda_{i+1}(\mathbf{A})$ for all $i$. For a real postive semidefinite (PSD) matrix $\mathbf{A}$ we use $\mathbf{A}^{\frac{1}{2}}$ to denote its symmetric square root. If $\mathbf{A}$ is invertible and PSD, then we use $\mathbf{A}^{-\frac{1}{2}}$ to denote the square root of $\mathbf{A}^{-1}$. The matrix $\mathbf{I}$ denotes the identity matrix (with dimension determined from the context, unless otherwise mentioned). For a vector $\mathbf{a}$, we use the notation $\mathbf{a} \geq \mathbf{0}$ to denote component-wise inequality; for a matrix $\mathbf{A}$, we use the notation $\mathbf{A} \succeq \mathbf{0}$ (or $\succ \mathbf{0}$ ) to denote that the matrix $\mathbf{A}$ is positive semidefinite (respectively, positive definite). We will assume all diagonals of $\mathbf{S}$ are strictly greater than zero. For a non-negative integer $m$ we denote $1, \ldots, m$ by $[m]$.

\section{Methodology}

We state a simple result (the proof of which is omitted) regarding the eigenvalues of the product of two matrices - a property that is used throughout the paper.

Proposition 1. For any two symmetric matrices $\mathbf{A}_{p \times p}$ and $\mathbf{B}_{p \times p}$, we have $\boldsymbol{\lambda}(\mathbf{A B})=\boldsymbol{\lambda}(\mathbf{B A})$.

A simple corollary of the above that is used widely in the paper is: 
Corollary 1. For any PSD matrix $\mathbf{\Phi}$, we have:

$$
\lambda\left(\boldsymbol{\Phi}^{\frac{1}{2}} \mathbf{S} \Phi^{\frac{1}{2}}\right)=\lambda(\mathbf{S} \boldsymbol{\Phi})=\lambda(\boldsymbol{\Phi S})=\lambda\left(\mathbf{S}^{\frac{1}{2}} \boldsymbol{\Phi} \mathbf{S}^{\frac{1}{2}}\right)
$$

\subsection{Reformulations}

In this section we present a reformulation of the low rank optimization Problem (2) to one that does not involve any (combinatorial) rank constraint: Proposition 2 reformulates Problem (2) as an optimization problem in $\boldsymbol{\Psi}$. The resulting problem (4) is amenable to efficient optimization techniques based on difference of convex optimization. In Proposition 3 we provide a characterization of an optimal solution of Problem (4).

Proposition 2. (a) Problem (2) is equivalent to:

$$
\begin{aligned}
& \operatorname{minimize}\left\{\log \operatorname{det}(\boldsymbol{\Psi})+\operatorname{tr}\left(\mathbf{S}^{*}\right)+\sum_{i=1}^{r}\left(\log \left(\max \left\{1, \lambda_{i}^{*}\right\}\right)-\max \left\{1, \lambda_{i}^{*}\right\}+1\right)\right\} \\
& \text { s.t. } \quad \boldsymbol{\Psi}=\operatorname{diag}\left(\psi_{1}, \ldots, \psi_{p}\right) \succeq \epsilon \mathbf{I},
\end{aligned}
$$

where, $\lambda_{1}^{*} \geq \lambda_{2}^{*} \geq \ldots \geq \lambda_{p}^{*}$ denote the eigenvalues of $\mathbf{S}^{*}:=\Psi^{-\frac{1}{2}} \mathbf{S} \Psi^{-\frac{1}{2}} ;$ and the optimization variables are $\mathbf{\Psi}, \mathbf{S}^{*}$ and $\left\{\lambda_{i}^{*}\right\}_{i \geq 1}$.

(b) Suppose $\hat{\mathbf{\Psi}}$ is a minimizer of Problem (4) and $\hat{\mathbf{L}}=\hat{\mathbf{\Psi}}^{\frac{1}{2}}\left[\hat{\mathbf{z}}_{1}, \hat{\mathbf{z}}_{2}, \ldots, \hat{\mathbf{z}}_{r}\right]$ where, $\hat{\mathbf{z}}_{1}, \hat{\mathbf{z}}_{2}, \ldots, \hat{\mathbf{z}}_{r}$ are eigenvectors of $\hat{\mathbf{\Psi}}^{-\frac{1}{2}} \mathbf{S} \hat{\mathbf{\Psi}}^{-\frac{1}{2}}$ corresponding to its top $r$ eigenvalues $\hat{\lambda}_{i}^{*}, i \leq r$ with $\left\|\hat{\mathbf{z}}_{i}\right\|^{2}=$ $\max \left\{1, \hat{\lambda}_{i}^{*}\right\}-1$ for all $i=1, \ldots, r$. Then $(\hat{\mathbf{\Psi}}, \hat{\mathbf{L}})$ is a minimizer of Problem (2).

Proof. Part (a): We first minimize Problem $\sqrt{2}$ with respect to $\mathbf{L}$ for a fixed value of $\boldsymbol{\Psi}$. A simple application of the Sherman Woodbury formula, with some rearrangement gives:

$$
\begin{aligned}
\boldsymbol{\Sigma}^{-1} & =\left(\boldsymbol{\Psi}+\mathbf{L} \mathbf{L}^{\top}\right)^{-1} \\
& =\boldsymbol{\Psi}^{-1}-\boldsymbol{\Psi}^{-1} \mathbf{L}\left(\mathbf{I}+\mathbf{L}^{\top} \boldsymbol{\Psi}^{-1} \mathbf{L}\right)^{-1} \mathbf{L}^{\top} \boldsymbol{\Psi}^{-1} \\
& =\boldsymbol{\Psi}^{-1}-\boldsymbol{\Psi}^{-\frac{1}{2}} \boldsymbol{\Psi}^{-\frac{1}{2}} \mathbf{L}\left(\mathbf{I}+\mathbf{L}^{\top} \boldsymbol{\Psi}^{-\frac{1}{2}} \boldsymbol{\Psi}^{-\frac{1}{2}} \mathbf{L}\right)^{-1} \mathbf{L}^{\top} \boldsymbol{\Psi}^{-\frac{1}{2}} \boldsymbol{\Psi}^{-\frac{1}{2}}
\end{aligned}
$$

Writing $\mathbf{L}^{*}=\Psi^{-\frac{1}{2}} \mathbf{L}$ in the last line of display $(5)$, we get:

$$
\boldsymbol{\Sigma}^{-1}=\boldsymbol{\Psi}^{-1}-\boldsymbol{\Psi}^{-\frac{1}{2}} \mathbf{L}^{*}\left(\mathbf{I}+\left(\mathbf{L}^{*}\right)^{\top} \mathbf{L}^{*}\right)^{-1}\left(\mathbf{L}^{*}\right)^{\top} \boldsymbol{\Psi}^{-\frac{1}{2}}
$$

The above implies that:

$$
\begin{aligned}
\operatorname{tr}\left(\boldsymbol{\Sigma}^{-1} \mathbf{S}\right) & =\operatorname{tr}\left(\boldsymbol{\Psi}^{-1} \mathbf{S}\right)-\operatorname{tr}\left(\boldsymbol{\Psi}^{-\frac{1}{2}} \mathbf{L}^{*}\left(\mathbf{I}+\left(\mathbf{L}^{*}\right)^{\top} \mathbf{L}^{*}\right)^{-1}\left(\mathbf{L}^{*}\right)^{\top} \boldsymbol{\Psi}^{-\frac{1}{2}} \mathbf{S}\right) \\
& =\operatorname{tr}\left(\boldsymbol{\Psi}^{-\frac{1}{2}} \mathbf{S} \boldsymbol{\Psi}^{-\frac{1}{2}}\right)-\operatorname{tr}\left(\left(\mathbf{L}^{*}\right)^{\top} \boldsymbol{\Psi}^{-\frac{1}{2}} \mathbf{S} \boldsymbol{\Psi}^{-\frac{1}{2}} \mathbf{L}^{*}\left(\mathbf{I}+\left(\mathbf{L}^{*}\right)^{\top} \mathbf{L}^{*}\right)^{-1}\right) \\
& =\operatorname{tr}\left(\mathbf{S}^{*}\right)-\operatorname{tr}\left(\left(\mathbf{L}^{*}\right)^{\top} \mathbf{S}^{*} \mathbf{L}^{*}\left(\mathbf{I}+\left(\mathbf{L}^{*}\right)^{\top} \mathbf{L}^{*}\right)^{-1}\right) \quad\left(\mathrm{Using}, \mathbf{S}^{*}=\boldsymbol{\Psi}^{-\frac{1}{2}} \mathbf{S} \boldsymbol{\Psi}^{-\frac{1}{2}}\right)
\end{aligned}
$$

where, in the second and third lines of display (6) we (repeatedly) used the fact that $\operatorname{tr}(A B)=$ $\operatorname{tr}(B A)$.

In addition, note that:

$$
\begin{aligned}
-\log \operatorname{det}\left(\boldsymbol{\Sigma}^{-1}\right)=\log \operatorname{det}(\boldsymbol{\Sigma}) & =\log \operatorname{det}\left(\mathbf{\Psi}+\mathbf{L} \mathbf{L}^{\top}\right) \\
& =\log \operatorname{det}(\boldsymbol{\Psi})+\log \operatorname{det}\left(\mathbf{I}+\mathbf{L}^{* \top} \mathbf{L}^{*}\right)
\end{aligned}
$$


Using (6), (7) in the objective function of Problem (2), i.e., $\mathcal{L}(\boldsymbol{\Sigma})=-\log \operatorname{det}\left(\boldsymbol{\Sigma}^{-1}\right)+\operatorname{tr}\left(\boldsymbol{\Sigma}^{-1} \mathbf{S}\right)$ we get the following equivalent reformulation of Problem (2):

$$
\begin{aligned}
& \text { minimize } h(\mathbf{\Psi}, \mathbf{L}):=\log \operatorname{det}(\mathbf{\Psi})+\log \operatorname{det}\left(\mathbf{I}+\mathbf{L}^{* \top} \mathbf{L}^{*}\right)+\operatorname{tr}\left(\mathbf{S}^{*}\right) \\
& -\operatorname{tr}\left(\mathbf{L}^{* \top} \mathbf{S}^{*} \mathbf{L}^{*}\left(\mathbf{I}+\mathbf{L}^{*^{\top}} \mathbf{L}^{*}\right)^{-1}\right) \\
& \text { s.t. } \quad \boldsymbol{\Psi}=\operatorname{diag}\left(\psi_{1}, \ldots, \psi_{p}\right) \succeq \epsilon \mathbf{I} \text {, }
\end{aligned}
$$

where, recall we use the notation: $\mathbf{L}^{*}=\Psi^{-\frac{1}{2}} \mathbf{L}$ and $\mathbf{S}^{*}=\Psi^{-\frac{1}{2}} \mathbf{S} \Psi^{-\frac{1}{2}}$; and the optimization variables in Problem (8) are $\boldsymbol{\Psi}, \mathbf{L}$ (and consequently, $\mathbf{L}^{*}, \mathbf{S}^{*}$ ). Note that $h(\boldsymbol{\Psi}, \mathbf{L})=h(\boldsymbol{\Psi}, \mathbf{L} \mathbf{U})$ for any orthogonal matrix $\mathbf{U}$. So we can substitute $\mathbf{L}$ by $\mathbf{L} \mathbf{U}$ in Problem (8). We choose $\mathbf{U}$ such that the columns of $\mathbf{L}^{*}$ are orthogonal or zero vectors. Note that the derivative of $h(\boldsymbol{\Psi}, \mathbf{L})=\log \operatorname{det}\left(\mathbf{L} \mathbf{L}^{\top}+\boldsymbol{\Psi}\right)+\operatorname{tr}\left(\left(\mathbf{L} \mathbf{L}^{\top}+\mathbf{\Psi}\right)^{-1} \mathbf{S}\right)$ w.r.t. $\mathbf{L}$ is given by:

$$
\frac{\partial h(\boldsymbol{\Psi}, \mathbf{L})}{\partial \mathbf{L}}=-2 \boldsymbol{\Sigma}^{-1}(\boldsymbol{\Sigma}-\mathbf{S}) \boldsymbol{\Sigma}^{-1} \mathbf{L} .
$$

Setting the above gradient to be zero and writing $\boldsymbol{\Sigma}=\mathbf{\Psi}+\mathbf{L} \mathbf{L}^{\top}$ we have $\mathbf{L}=\mathbf{S}\left(\mathbf{\Psi}+\mathbf{L} \mathbf{L}^{\top}\right)^{-1} \mathbf{L}$. After some elementary algebra this can be written as:

$$
\mathbf{S}^{*} \mathbf{L}^{*}\left(\mathbf{I}+\mathbf{L}^{*^{\top}} \mathbf{L}^{*}\right)^{-1}=\mathbf{L}^{*}
$$

Since we choose the columns of $\mathbf{L}^{*}$ to be pairwise orthogonal or zero vectors, it follows that $\mathbf{I}+\mathbf{L}^{* \top} \mathbf{L}^{*}$ is a diagonal matrix. This means that 10$]$ is a collection of eigenvector equations for the matrix $\mathbf{S}^{*}$. From condition 10 we have that at an optimal value of $\mathbf{L}$, the columns of $\mathbf{L}^{*}$ are either pairwise orthogonal eigenvectors of $\mathbf{S}^{*}$ with eigenvalues as the diagonal entries of $\mathbf{I}+\mathbf{L}^{* \top} \mathbf{L}^{*}$ or they are zero vectors. Denoting the columns of $\mathbf{L}^{*}$ by $\mathbf{z}_{i}, i=1, \ldots, r$ the part of the function $h(\mathbf{\Psi}, \mathbf{L})$ in display (8), that depends upon $\mathbf{L}$ is given by:

$$
g(\mathbf{L})=\sum_{i=1}^{r}\left(\log \left(1+\mathbf{z}_{i}^{\top} \mathbf{z}_{i}\right)-\frac{\mathbf{z}_{i}^{\top} \mathbf{S}^{*} \mathbf{z}_{i}}{1+\mathbf{z}_{i}^{\top} \mathbf{z}_{i}}\right) .
$$

Since $\mathbf{z}_{i}$ s are pairwise orthogonal or zero vectors, it follows from equation 10 that

$$
\mathbf{S}^{*} \mathbf{z}_{i}=\beta_{i} \mathbf{z}_{i}, \text { with } \beta_{i}=1+\mathbf{z}_{i}^{\top} \mathbf{z}_{i} .
$$

Note that in the above equation, either $\beta_{i}=1$ with $\mathbf{z}_{i}=0$ or $\beta_{i}>1$ and $\beta_{i}$ equals some eigenvalue of $\mathbf{S}^{*}$ with eigenvector $\mathbf{z}_{i}$ - thus (11) becomes

$$
g(\mathbf{L})=\sum_{i=1}^{r}\left(\log \left(\beta_{i}\right)-\beta_{i}+1\right) .
$$

Note that $\beta \mapsto \log (\beta)-\beta+1$ is strictly decreasing for all $\beta \geq 1$. So it is easy to see that (13) is minimized for $\beta_{i}=\max \left\{1, \lambda_{i}^{*}\right\}$ for $1 \leq i \leq r$, where, $\lambda_{1}^{*} \geq \ldots \geq \lambda_{r}^{*}$ are the top $r$ eigenvalues of $\mathbf{S}^{*}$. The optimal choice of $\mathbf{z}_{i}$ is given by $\mathbf{z}_{i}=0$ when $\beta_{i}=1$ and when $\beta_{i}>1, \mathbf{z}_{i}$ is an eigenvector of $\mathbf{S}^{*}$ with eigenvalue $\lambda_{i}^{*}$ and with $\mathbf{z}_{i}^{\top} \mathbf{z}_{i}=\max \left\{1, \lambda_{i}^{*}\right\}-1$. Noting that

$$
\min _{\mathbf{\Psi} \succeq \in \mathbf{I}, \mathbf{L}} h(\mathbf{\Psi}, \mathbf{L})=\min _{\mathbf{\Psi} \succeq \in \mathbf{I}}\left\{\min _{\mathbf{L}} h(\mathbf{\Psi}, \mathbf{L})\right\},
$$

and substituting the values of $\mathbf{L}$ that minimize the inner minimization problem, above, into the objective function $h(\boldsymbol{\Psi}, \mathbf{L})$, we obtain (4).

Part (b): The proof of this part is a consequence of the proof of Part (a). 
The method of minimizing the objective function w.r.t. $\mathbf{L}$ with $\boldsymbol{\Psi}$ held fixed, is inspired by the classical work of [19, 20, 17] - this line of work however, assumes $\mathbf{S}$ to be of full rank. We do not assume that $\mathbf{S}$ is full rank. 28] investigate the existence of ML solutions for a general $\mathbf{S}-$ no algorithms for computing the solution are presented. The expression (4) derived herein, does not appear in [28. Formulation (4) plays a key role in developing algorithms for Problem (4), a main focus of this paper.

Proposition 3 shows that any solution of Problem (4) is bounded above.

Proposition 3. Let $\hat{\boldsymbol{\Psi}}$ be a solution of Problem (4). Then $\operatorname{diag}(\mathbf{S}) \succeq \hat{\mathbf{\Psi}} \succeq \epsilon \mathbf{I}$.

Proof. Note that for any $\boldsymbol{\Psi} \succ \mathbf{0}, \frac{\partial h}{\partial \boldsymbol{\Psi}}=\operatorname{diag}\left(\boldsymbol{\Sigma}^{-1}(\boldsymbol{\Sigma}-\mathbf{S}) \boldsymbol{\Sigma}^{-1}\right)$. Let $\mathbf{L}(\boldsymbol{\Psi})$ be the optimal value of $\mathbf{L}$ for fixed value of $\boldsymbol{\Psi}$. Then from the proof of Proposition 2 it follows that $\frac{\partial h}{\partial \mathbf{L}}=0$ at $(\boldsymbol{\Psi}, \mathbf{L}(\boldsymbol{\Psi}))$. Setting $(9)$ to zero, we have $\mathbf{L}=\mathbf{S} \boldsymbol{\Sigma}^{-1} \mathbf{L}$; and applying Sherman Woodbury formula on $\left(\mathbf{\Psi}+\mathbf{L} \mathbf{L}^{\top}\right)^{-1}$, we have the following chain of inequalities:

$$
\begin{aligned}
\mathbf{L} & =\mathbf{S} \boldsymbol{\Sigma}^{-1} \mathbf{L} \\
& =\mathbf{S}\left(\boldsymbol{\Psi}^{-1}-\boldsymbol{\Psi}^{-1} \mathbf{L}\left(\mathbf{I}+\mathbf{L}^{\top} \boldsymbol{\Psi}^{-1} \mathbf{L}\right)^{-1} \mathbf{L}^{\top} \boldsymbol{\Psi}^{-1}\right) \mathbf{L} \\
& =\mathbf{S} \boldsymbol{\Psi}^{-1} \mathbf{L}-\mathbf{S} \boldsymbol{\Psi}^{-1} \mathbf{L}\left(\left(\mathbf{I}+\mathbf{L}^{\top} \boldsymbol{\Psi}^{-1} \mathbf{L}\right)^{-1} \mathbf{L}^{\top} \boldsymbol{\Psi}^{-1} \mathbf{L}\right) \\
& =\mathbf{S} \boldsymbol{\Psi}^{-1} \mathbf{L}-\mathbf{S} \boldsymbol{\Psi}^{-1} \mathbf{L}\left(\mathbf{I}-\left(\mathbf{I}+\mathbf{L}^{\top} \boldsymbol{\Psi}^{-1} \mathbf{L}\right)^{-1}\right) \\
& =\mathbf{S} \boldsymbol{\Psi}^{-1} \mathbf{L}\left(\mathbf{I}+\mathbf{L}^{\top} \boldsymbol{\Psi}^{-1} \mathbf{L}\right)^{-1} .
\end{aligned}
$$

Eqn (15) follows from (5); Eqn (17) follows from 116 by using the observation that for a PSD matrix $\mathbf{B}$ we have the following identity: $(\mathbf{I}+\mathbf{B})^{-1} \mathbf{B}=\mathbf{I}-(\mathbf{I}+\mathbf{B})^{-1}$ (which can be verified by simple algebra).

Moreover using (5) on $\boldsymbol{\Sigma}^{-1}$ the expression $\mathbf{S} \boldsymbol{\Sigma}^{-1}$ simplifies as:

$$
\begin{aligned}
\mathbf{S} \boldsymbol{\Sigma}^{-1} & =\mathbf{S} \boldsymbol{\Psi}^{-1}-\left(\mathbf{S} \boldsymbol{\Psi}^{-1} \mathbf{L}\left(\mathbf{I}+\mathbf{L}^{\top} \boldsymbol{\Psi}^{-1} \mathbf{L}\right)^{-1}\right)\left(\mathbf{L}^{\top} \boldsymbol{\Psi}^{-1}\right) \\
& =\mathbf{S} \boldsymbol{\Psi}^{-1}-\mathbf{L} \mathbf{L}^{\top} \boldsymbol{\Psi}^{-1} \quad(\text { Using expression of } \mathbf{L} \text { from rhs of } 18) \\
& =\mathbf{S} \boldsymbol{\Psi}^{-1}-(\boldsymbol{\Sigma}-\boldsymbol{\Psi}) \boldsymbol{\Psi}^{-1} \quad\left(\text { Since, } \boldsymbol{\Sigma}=\mathbf{L} \mathbf{L}^{\top}+\mathbf{\Psi}\right) \\
& =\mathbf{S} \boldsymbol{\Psi}^{-1}-\mathbf{\Sigma} \boldsymbol{\Psi}^{-1}+\mathbf{I} .
\end{aligned}
$$

Note that we have the following expression for $\boldsymbol{\Sigma}^{-1}(\boldsymbol{\Sigma}-\mathbf{S}) \boldsymbol{\Sigma}^{-1}$ :

$$
\begin{aligned}
\boldsymbol{\Sigma}^{-1}(\boldsymbol{\Sigma}-\mathbf{S}) \boldsymbol{\Sigma}^{-1} & =\boldsymbol{\Sigma}^{-1}-\boldsymbol{\Sigma}^{-1}\left(\mathbf{S} \boldsymbol{\Sigma}^{-1}\right) \\
& =\boldsymbol{\Sigma}^{-1}-\boldsymbol{\Sigma}^{-1}\left(\mathbf{S} \boldsymbol{\Psi}^{-1}-\boldsymbol{\Sigma} \boldsymbol{\Psi}^{-1}+\mathbf{I}\right) \\
& =-\left(\boldsymbol{\Sigma}^{-1} \mathbf{S}\right) \boldsymbol{\Psi}^{-1}+\mathbf{\Psi}^{-1} \\
& =-\left(\boldsymbol{\Psi}^{-1} \mathbf{S}-\boldsymbol{\Psi}^{-1} \boldsymbol{\Sigma}+\mathbf{I}\right) \boldsymbol{\Psi}^{-1}+\boldsymbol{\Psi}^{-1} \\
& =\boldsymbol{\Psi}^{-1}(\boldsymbol{\Sigma}-\mathbf{S}) \boldsymbol{\Psi}^{-1}
\end{aligned}
$$

where, the second line follows by using expression of $\mathbf{S} \boldsymbol{\Sigma}^{-1}$ from $(19)$; and the fourth line follows by using the same expression for $\boldsymbol{\Sigma}^{-1} \mathbf{S}=\left(\mathbf{S} \boldsymbol{\Sigma}^{-1}\right)^{\top}$.

Using [20, the expression for $\frac{\partial h}{\partial \boldsymbol{\Psi}}=\boldsymbol{\Sigma}^{-1}(\boldsymbol{\Sigma}-\mathbf{S}) \boldsymbol{\Sigma}^{-1}$ reduces to

$$
\frac{\partial h}{\partial \boldsymbol{\Psi}}=\operatorname{diag}\left(\boldsymbol{\Sigma}^{-1}(\boldsymbol{\Sigma}-\mathbf{S}) \boldsymbol{\Sigma}^{-1}\right)=\boldsymbol{\Psi}^{-1} \operatorname{diag}(\boldsymbol{\Sigma}-\mathbf{S}) \boldsymbol{\Psi}^{-1} .
$$

So, we conclude that $\frac{\partial h}{\partial \psi_{i}}>0$ if $\psi_{i}>s_{i i}$ for some $1 \leq i \leq p$. We are minimizing Problem (4), so the optimal solution must satisfy $\hat{\psi}_{i} \leq s_{i i}$. This completes the proof. 
We present another equivalent representation of Problem (4), by a simple change of variables $\boldsymbol{\Phi}:=\boldsymbol{\Psi}^{-1}$. In what follows below, unless otherwise specified, we use the shorthand $\boldsymbol{\Phi}:=$ $\operatorname{diag}\left(\phi_{1}, \ldots, \phi_{p}\right)$ and $\phi=\left(\phi_{1}, \ldots, \phi_{p}\right)$.

Corollary 2. Problem (4) is equivalent to the following optimization problem in $\phi$ :

$$
\begin{aligned}
\operatorname{minimize} & f(\phi):=\sum_{i=1}^{p}\left(-\log \phi_{i}+s_{i i} \phi_{i}\right)+\sum_{i=1}^{r}\left(\log \left(\max \left\{1, \lambda_{i}^{*}\right\}\right)-\max \left\{1, \lambda_{i}^{*}\right\}+1\right) \\
\text { s.t. } \quad & \mathbf{0} \prec \boldsymbol{\Phi}=\operatorname{diag}\left(\phi_{1}, \ldots, \phi_{p}\right) \preceq \frac{1}{\epsilon} \mathbf{I},
\end{aligned}
$$

where, $\left\{\lambda^{*}\right\}_{1}^{r}$ are the top $r$ eigenvalues of $\mathbf{S}^{*}=\boldsymbol{\Phi}^{\frac{1}{2}} \mathbf{S} \Phi^{\frac{1}{2}}$. If $\hat{\boldsymbol{\Psi}}$ is a solution to Problem (4) then, we have $\hat{\mathbf{\Psi}}=\hat{\boldsymbol{\Phi}}^{-1}$, where, $\hat{\boldsymbol{\Phi}}$ is a solution to Problem 22 .

Remark 1. Problem (4) (and Problem (22) is a minimization problem in $\boldsymbol{\Psi}$ (respectively, $\mathbf{\Phi}$ ), unlike the (rank constrained) Problem (2) with variables $\mathbf{L}$ and $\boldsymbol{\Psi}$. Note that Problem (22) is nonconvex due to the nonconvex objective function, though the constraints are convex. Corollary 3 shows that the the objective function $f(\phi)$ appearing in Problem (22) is neither convex nor concave but it can be written as a difference of simple convex functions.

\subsection{Expressing Problem (22) as a difference of convex functions}

In this section we show via Proposition 4 and 5 that the objective function in Problem $(22)$ can be written as a difference of two convex functions (Corollary 3). This renders the application of algorithms based on difference of convex optimization, to get good solutions to Problem (22). Proposition 6 shows that when the sample covariance matrix $\mathbf{S}$ is full rank, the objective function in Problem (22) can be expressed purely in terms of the eigenvalues of $\mathbf{S}^{*}$.

Let $y_{(1)} \geq \ldots \geq y_{(p)}$ be an ordering of $\left\{y_{i}\right\}_{1}^{p} \in[0, \infty)^{p}$ and define:

$$
H_{r}(\mathbf{y}):=\sum_{i=1}^{r}\left(\log \left(\max \left\{1, y_{(i)}\right\}\right)-\max \left\{1, y_{(i)}\right\}+1\right) .
$$

The following proposition shows that $\mathbf{y} \mapsto H_{r}(\mathbf{y})$ is concave on $\mathbf{y} \geq \mathbf{0}$.

Proposition 4. $H_{r}(\mathbf{y})$ as defined in $(23)$, is concave on $\mathbf{y} \geq \mathbf{0}$.

Proof. We first establish that $H_{r}(\mathbf{y})$ admits the following representation:

$$
\begin{gathered}
H_{r}(\mathbf{y})=\min _{\mathbf{w}} \quad \widetilde{H}(\mathbf{w} ; \mathbf{y}):=\sum_{i=1}^{p} w_{i}\left(\log \left(\max \left\{1, y_{i}\right\}\right)-\max \left\{1, y_{i}\right\}+1\right) \\
\text { s.t. } \quad \sum_{i=1}^{p} w_{i}=r, \quad 0 \leq w_{i} \leq 1, i \in[p],
\end{gathered}
$$

as the minimum (w.r.t. the optimization variable $\mathbf{w}$ ) of the linear functional $\mathbf{w} \mapsto \widetilde{H}(\mathbf{w} ; \mathbf{y})$. To see why this is true, note that the scalar function $y \mapsto \log (\max \{1, y\})-\max \{1, y\}+1$ is decreasing on $y \geq 0$. Hence the $\operatorname{sum} \sum_{i=1}^{p} w_{i}\left(\log \left(\max \left\{1, y_{i}\right\}\right)-\max \left\{1, y_{i}\right\}+1\right)$ will be minimized for a choice: $w_{i}=1$ whenever $y_{i}$ is one of the top $r$ elements among $y_{1}, \ldots, y_{p}$; and $w_{i}=0$ for all other choices of $i$. This justifies representation (24).

For any $\Re \ni y \geq 0$, note that $y \mapsto \log (\max \{1, y\})-\max \{1, y\}+1$ is concave. Hence, for every fixed $\Re^{p} \ni \mathbf{w} \geq \mathbf{0}$, the function

$$
\left(y_{1}, \ldots, y_{p}\right) \mapsto \sum_{i=1}^{p} w_{i}\left(\log \left(\max \left\{1, y_{i}\right\}\right)-\max \left\{1, y_{i}\right\}+1\right)
$$


is concave on $\mathbf{y} \geq \mathbf{0}$. Since the pointwise infimum of a family of concave functions is concave [10], $H_{r}(\mathbf{y})$ is concave on the nonnegative reals.

Proposition 5. For any $r \in[p]$, the function

$$
\phi \mapsto h(\phi):=\sum_{i=1}^{r}\left(\log \left(\max \left\{1, \lambda_{i}^{*}\right\}\right)-\max \left\{1, \lambda_{i}^{*}\right\}+1\right)
$$

is concave on $\boldsymbol{\phi} \geq \mathbf{0}$; where, $\left\{\lambda_{i}^{*}\right\}_{1}^{p}$ are the eigenvalues of $\mathbf{S}^{*}$.

Proof. Note $\boldsymbol{\lambda}\left(\mathbf{S}^{*}\right)=\boldsymbol{\lambda}\left(\mathbf{S}^{\frac{1}{2}} \mathbf{\Phi} \mathbf{S}^{\frac{1}{2}}\right)$ (Corollary 1). By a classic result due to Davis [12, 23, the following mapping

$$
\mathbf{S}^{\frac{1}{2}} \mathbf{\Phi} \mathbf{S}^{\frac{1}{2}} \mapsto \sum_{i=1}^{r}\left(\log \left(\max \left\{1, \lambda_{i}^{*}\right\}\right)-\max \left\{1, \lambda_{i}^{*}\right\}+1\right)
$$

is concave in $\mathbf{S}^{\frac{1}{2}} \mathbf{\Phi} \mathbf{S}^{\frac{1}{2}}$ if and only if the function $(23)$ is symmetri $3^{3}$ and concave in $\mathbf{y}$ on $\mathbf{y} \geq \mathbf{0}$. It is easy to see that the function in (23) is symmetric and concavity follows from Proposition 4 . So we conclude that the map in $(26)$ is concave in $\mathbf{S}^{\frac{1}{2}} \mathbf{\Phi} \mathbf{S}^{\frac{1}{2}}$. The linearity of the map $\phi \mapsto \mathbf{S}^{\frac{1}{2}} \mathbf{\Phi} \mathbf{S}^{\frac{1}{2}}$ implies that $h(\boldsymbol{\phi})$ is concave in $\boldsymbol{\phi}$ on $\boldsymbol{\phi} \geq \mathbf{0}$. This completes the proof of the proposition.

Corollary 3. For any $\phi>\mathbf{0}, f(\phi)$ can be written as the difference of two convex functions, $f_{i}(\phi), i=1,2$ that is: $f(\phi)=f_{1}(\phi)-f_{2}(\phi)$, where,

$$
f_{1}(\phi)=\sum_{i=1}^{p}\left(-\log \phi_{i}+s_{i i} \phi_{i}\right) \quad \text { and } \quad f_{2}(\phi)=-\sum_{i=1}^{r}\left(\log \left(\max \left\{1, \lambda_{i}^{*}\right\}\right)-\max \left\{1, \lambda_{i}^{*}\right\}+1\right) .
$$

Proof. The convexity of $f_{1}(\phi)$ is easy to see. Proposition 5 implies that $f_{2}(\phi)$ is convex.

When $\mathbf{S}$ is full rank, i.e., $\mathbf{S} \succ \mathbf{0}$, then Problem 22 can be rewritten purely as a function of the eigenvalues $\left\{\lambda_{i}^{*}\right\}_{i \geq 1}$ - this is established in Proposition 6 .

Proposition 6. If $\mathbf{S} \succ \mathbf{0}$ Problem 22 is equivalent to the following problem:

$$
\begin{array}{ll}
\operatorname{minimize} & \bar{f}(\boldsymbol{\phi}):=\sum_{i=1}^{p}\left(-\log \lambda_{i}^{*}+\lambda_{i}^{*}\right)+\sum_{i=1}^{r}\left(\log \left(\max \left\{1, \lambda_{i}^{*}\right\}\right)-\max \left\{1, \lambda_{i}^{*}\right\}+1\right) \\
\text { s.t. } & \mathbf{0} \prec \mathbf{\Phi}=\operatorname{diag}\left(\phi_{1}, \ldots, \phi_{p}\right) \preceq \frac{1}{\epsilon} \mathbf{I},
\end{array}
$$

where, $\left\{\lambda_{i}^{*}\right\}_{1}^{p}$ are the eigenvalues of $\boldsymbol{\Phi}^{\frac{1}{2}} \mathbf{S} \Phi^{\frac{1}{2}}$.

Proof. Problem 222 is equivalent to minimizing $\bar{f}(\boldsymbol{\phi}):=-\log \operatorname{det}(\mathbf{S})+f(\boldsymbol{\phi})$ over $\mathbf{0} \prec \mathbf{\Phi} \preceq \frac{1}{\epsilon} \mathbf{I}$. The function $\bar{f}(\phi)$ can be expressed as:

$$
\begin{aligned}
\bar{f}(\boldsymbol{\phi}) & =\sum_{i=1}^{p}\left(-\log \phi_{i}+s_{i i} \phi_{i}\right)-\log \operatorname{det}(\mathbf{S})+\sum_{i=1}^{r}\left(\log \left(\max \left\{1, \lambda_{i}^{*}\right\}\right)-\max \left\{1, \lambda_{i}^{*}\right\}+1\right) \\
& =-\log \operatorname{det}\left(\mathbf{S}^{*}\right)+\operatorname{tr}\left(\mathbf{S}^{*}\right)+\sum_{i=1}^{r}\left(\log \left(\max \left\{1, \lambda_{i}^{*}\right\}\right)-\max \left\{1, \lambda_{i}^{*}\right\}+1\right) \\
& =\sum_{i=1}^{p}\left(-\log \lambda_{i}^{*}+\lambda_{i}^{*}\right)+\sum_{i=1}^{r}\left(\log \left(\max \left\{1, \lambda_{i}^{*}\right\}\right)-\max \left\{1, \lambda_{i}^{*}\right\}+1\right)
\end{aligned}
$$

\footnotetext{
${ }^{3} \mathrm{~A}$ function $g\left(y_{1}, \ldots, y_{p}\right): \Re^{p} \rightarrow \Re$ is said to be symmetric in its arguments if, for any permutation $\pi$ of the indices $\{1, \ldots, p\}$, we have $g\left(y_{1}, \ldots, y_{p}\right)=g\left(y_{\pi(1)}, \ldots, y_{\pi(p)}\right)$
} 
where, line 29 follows from 28 by observing that

$$
\begin{aligned}
\sum_{i=1}^{p}\left(-\log \left(\phi_{i}\right)+s_{i i} \phi_{i}\right)-\log \operatorname{det}(\mathbf{S}) & =-\log \operatorname{det}(\mathbf{\Phi})+\operatorname{tr}(\mathbf{S} \boldsymbol{\Phi})-\log \operatorname{det}(\mathbf{S}) \\
& =-\log \operatorname{det}(\mathbf{S} \boldsymbol{\Phi})+\operatorname{tr}(\mathbf{S} \boldsymbol{\Phi}) \\
& =-\log \operatorname{det}\left(\mathbf{S}^{*}\right)+\operatorname{tr}\left(\mathbf{S}^{*}\right)
\end{aligned}
$$

where, $\mathbf{S}^{*}=\boldsymbol{\Phi}^{\frac{1}{2}} \mathbf{S} \Phi^{\frac{1}{2}}$; and the last equality in 31 made use of Corollary 1 Moreover, note that since $\mathbf{S}$ is of full rank and $\boldsymbol{\Phi} \succ \mathbf{0}$; all the eigenvalues $\lambda_{i}^{*}>0$. This completes the proof.

Proposition 6 provides an interesting alternate characterization of the formulation presented in Corollary 3 - this helps us gain additional understanding of the optimization problem for ML factor analysis when $\mathbf{S} \succ \mathbf{0}$.

\subsubsection{Computing subgradients}

Herein, we study the computation of (sub)gradients 29] of properties of the functions $f_{1}(\phi)$ and $f_{2}(\phi)$. Note that $f_{1}(\phi)$ is differentiable. However, the convex (spectral) function $f_{2}(\cdot)$ is not differentiable. A subgradient of $f_{2}(\phi)$ can be computed following the work of [22] on differentiability of spectral functions of Hermitian matrices. To this end, consider the representation of $H_{r}(\mathbf{y})$ in 24$)$; and define the function $g(y)=\log (\max \{1, y\})-\max \{1, y\}+1$ on $y \geq 0$. Define $\widetilde{H}_{r}(\mathbf{y})=-H_{r}(\mathbf{y})$ which is a convex function in $\mathbf{y}$. If $\partial \widetilde{H}_{r}(\mathbf{y})$ is a subgradient of $\widetilde{H}_{r}(\mathbf{y})$, then it can be computed by using Danskin's theorem: $\partial \widetilde{H}_{r}(\mathbf{y})=-\sum_{i=1}^{p} \hat{w}_{i} \nabla g\left(y_{i}\right)$, where, $\hat{\mathbf{w}}$ is a minimizer of the inner optimization task in Problem (24); and $\nabla g\left(y_{i}\right) \in \Re^{p}$ is the gradient of $g\left(y_{i}\right)$, with $i$ th coordinate given by $\nabla_{i} g\left(y_{i}\right)=\min \left\{0, \frac{1}{y_{i}}-1\right\}$, and $\nabla_{j} g\left(y_{i}\right)=0$ for all $j \neq i$. The function $\widetilde{H}_{r}(\mathbf{y})$ is differentiable at $\mathbf{y}$ iff $\hat{\mathbf{w}}$ is unique 4 . The set of all subgradients of $\widetilde{H}_{r}(\mathbf{y})$ is given by

$$
\operatorname{Conv}\left(\left\{-\sum_{i=1}^{p} \hat{w}_{i} \nabla g\left(y_{i}\right): \hat{\mathbf{w}} \text { is a minimizer of Problem 24 }\right\}\right)
$$

Let us consider a matrix $\mathbf{A} \succeq \mathbf{0}$, with eigen decomposition $\mathbf{A}=\mathbf{V} \operatorname{diag}(\boldsymbol{\lambda}) \mathbf{V}^{\top}$, and consequently consider the spectral convex function $\tilde{g}_{r}(\mathbf{A}):=-\sum_{i=1}^{r} g\left(\lambda_{i}\right)$. Using properties of subgradients of spectral functions [22], we have that a subgradient of $\mathbf{A} \mapsto \tilde{g}_{r}(\mathbf{A})$ is given by:

$$
\partial \tilde{g}_{r}(\mathbf{A})=\mathbf{V} \operatorname{diag}\left(\partial \widetilde{H}_{r}(\boldsymbol{\lambda})\right) \mathbf{V}^{\top}
$$

where, $\partial \widetilde{H}_{r}(\boldsymbol{\lambda})$ is a subgradient of $\boldsymbol{\lambda} \mapsto \widetilde{H}_{r}(\boldsymbol{\lambda})$.

If $\boldsymbol{\Phi}^{\frac{1}{2}} \mathbf{S} \boldsymbol{\Phi}^{\frac{1}{2}}=\mathbf{U} \operatorname{diag}\left(\lambda_{1}^{*}, \ldots, \lambda_{p}^{*}\right) \mathbf{U}^{\top}$ is the eigen decomposition of $\boldsymbol{\Phi}^{\frac{1}{2}} \mathbf{S} \boldsymbol{\Phi}^{\frac{1}{2}}$; then using the chain rule, the $i$ th coordinate of $\partial f_{2}(\phi)$ is given by:

$$
\partial_{i} f_{2}(\phi)=\left(\boldsymbol{\Phi}^{-\frac{1}{2}} \mathbf{U D}_{1} \mathbf{U}^{\top} \boldsymbol{\Phi}^{\frac{1}{2}} \mathbf{S}\right)_{i i}
$$

where, $\mathbf{D}_{1}=\operatorname{diag}\left(\delta_{1}, \ldots, \delta_{p}\right)$, with

$$
\delta_{i}= \begin{cases}\max \left\{0,1-\frac{1}{\lambda_{i}^{*}}\right\} & \text { if } 1 \leq i \leq r \\ 0 & \text { otherwise }\end{cases}
$$

\footnotetext{
${ }^{4}$ We note that this occurs if $g\left(y_{(r+1)}\right)=g\left(y_{(r)}\right)$.
} 


\subsection{Algorithm for Problem (22): A DC optimization approach}

Problem 22 is a nonconvex optimization problem with semidefinite constraints and obtaining a global minimum for a general $r$ is quite challenging. We thus focus on developing efficient computational procedures for obtaining good (feasible) solutions to Problem (22). By Corollary 3 . Problem 22 is equivalent to the following nonconvex optimization problem:

$$
\begin{aligned}
\operatorname{minimize} & f(\phi)=f_{1}(\phi)-f_{2}(\phi) \\
\text { s.t. } & \phi \in \mathcal{C}:=\left\{\phi: \frac{1}{\epsilon} \geq \phi_{i}>0, i \in[p]\right\} .
\end{aligned}
$$

We use a sequential linearization procedure: wherein, at every iteration, we linearize the function $f_{2}(\phi)$ (leaving $f_{1}(\cdot)$ as is) and solve the resultant convex problem. This is an instance of the well-known difference of convex optimization based algorithms [16, 35, 27, or DC algorithms in short. In the machine learning community, these methods are also known as the convex concave procedure [36, 37]. These algorithms have gained significant traction in the wider optimization community, especially recently, due to their pervasive use in practice - some excellent recent works on this topic include [1, 13, 25, 26] (see also references therein). However, to our knowledge, ours is the first paper to use this approach in the context of ML factor analysis. We will also like to remind the reader that the reformulations in Section 2.1 play a key role in arriving at decomposition (34) - this sets the stage for the application of the DC approach.

Let us formally describe the algorithm. If $\phi^{(k)} \in \mathcal{C}$ denotes the value of $\phi$ at the $k$ th iteration, we linearize $f_{2}(\phi)$ at $\phi^{(k)}$ with $f_{1}(\phi)$ unchanged and obtain a convex approximation of $f(\phi)$, denoted by $F\left(\phi ; \phi^{(k)}\right)$; and this is given by:

$$
f(\phi) \approx f_{1}(\phi)-\left(f_{2}\left(\phi^{(k)}\right)+\left\langle\nabla_{k}, \phi-\phi^{(k)}\right\rangle\right):=F\left(\phi ; \phi^{(k)}\right)
$$

where, $\nabla_{k}$ is a subgradient of $f_{2}(\phi)$ at $\phi^{(k)}$ (Section 2.2.1). We compute $\phi^{(k+1)}$ as:

$$
\boldsymbol{\phi}^{(k+1)} \underset{\frac{1}{\epsilon} \mathbf{1} \geq \boldsymbol{\phi}>\mathbf{0}}{\arg \min } F\left(\boldsymbol{\phi} ; \boldsymbol{\phi}^{(k)}\right)=\underset{\frac{1}{\epsilon} \mathbf{1} \geq \boldsymbol{\phi}>\mathbf{0}}{\arg \min } \sum_{i=1}^{p}\left(-\log \phi_{i}+s_{i i} \phi_{i}-\nabla_{k, i} \phi_{i}\right),
$$

where, $\nabla_{k, i}$ is the $i$ th coordinate of $\nabla_{k} \in \Re^{p}$ (for all $i \in[p]$ ). The $i$ th entry of $\boldsymbol{\phi}^{(k+1)}$ is given by:

$$
\phi_{i}^{(k+1)}=\min \left\{\frac{1}{s_{i i}-\nabla_{k, i}}, \frac{1}{\epsilon}\right\} \quad \text { for } \quad i \in[p] .
$$

The updates continue till some stopping criterion is satisfied. This can be in terms of the relative change in the successive objective values: $f\left(\phi^{(k)}\right)-f\left(\phi^{(k+1)}\right)<\eta\left|f\left(\phi^{(k+1)}\right)\right|$ or relative changes in successive iterate values: $\left\|\phi^{(k+1)}-\phi^{(k)}\right\|_{2}<\eta\left\|\phi^{(k)}\right\|_{2}$; where, $\eta>0$ denotes a pre-specified tolerance level. We summarize the algorithm in the following display for convenience.

Algorithm 1: An algorithm for Problem (34).

Initialize with $\phi^{(1)} \in \mathcal{C}$ and update $\phi^{(k)}$ using 36 until some stopping criterion like $f\left(\phi^{(k)}\right)-f\left(\phi^{(k+1)}\right)<\eta\left|f\left(\phi^{(k+1)}\right)\right|$ is met.

\subsection{Computational guarantees for Algorithm 1}

We present herein, computational guarantees for Algorithm 1 in terms of the number of iterations required to deliver an approximate first order stationary point; and asymptotic convergence to a first order stationary point. Towards this end, we recall certain standard definitions of first 
order stationary conditions for Problem (34) (see for example, [27]). $\tilde{\phi} \in \mathcal{C}$ is said to be a first order stationary point of Problem (34) if the following condition holds:

$$
\begin{aligned}
& \tilde{\boldsymbol{\phi}} \in \underset{\boldsymbol{\phi}}{\arg \min } F(\boldsymbol{\phi} ; \tilde{\boldsymbol{\phi}})=f_{1}(\boldsymbol{\phi})-\left\langle\partial f_{2}(\tilde{\boldsymbol{\phi}}), \boldsymbol{\phi}-\tilde{\boldsymbol{\phi}}\right\rangle \\
& \text { s.t. } \quad \boldsymbol{\phi} \in \mathcal{C}=\left\{\boldsymbol{\phi}: \frac{1}{\epsilon} \mathbf{1} \geq \boldsymbol{\phi}>\mathbf{0}\right\},
\end{aligned}
$$

for some choice of a subgradient $\partial f_{2}(\tilde{\phi})$. From standard optimality conditions of convex functions [29, 9, the above condition is equivalent to saying that, there exists a subgradient $\partial f_{2}(\tilde{\phi})$ such that:

$$
\partial f_{2}(\tilde{\boldsymbol{\phi}}) \in \nabla f_{1}(\tilde{\boldsymbol{\phi}})+\mathcal{N}(\tilde{\boldsymbol{\phi}} ; \mathcal{C})
$$

where, $\mathcal{N}(\tilde{\boldsymbol{\phi}} ; \mathcal{C})$ is the normal cone to the convex set $\mathcal{C}$ at the point $\tilde{\boldsymbol{\phi}}$. Recall that $\mathcal{N}(\tilde{\boldsymbol{\phi}} ; \mathcal{C})$ is the convex cone of all vectors $\mathbf{d} \in \Re^{p}$ such that $\langle\mathbf{d}, \phi-\tilde{\phi}\rangle \geq 0$ for all $\phi \in \mathcal{C}$. In (38) the right hand side denotes the standard Minkowski sum of a vector $\left(\nabla f_{1}(\tilde{\phi})\right)$ and a set $(\mathcal{N}(\phi ; \mathcal{C}))$.

Proposition 7 shows that the sequence $\phi^{(k)}$ leads to a decreasing sequence of objective values, where the amount of decrease is lower bounded by the squared norm of successive difference of the iterates $\left\{\phi^{(k)}\right\}$.

Proposition 7. Let $\phi^{(k)}$ be a sequence generated via Algorithm 1. Then, there exists $\rho \geq \epsilon^{2}$ such that for every $k \geq 1$ :

$$
f\left(\phi^{(k)}\right)-f\left(\phi^{(k+1)}\right) \geq \frac{\rho}{2}\left\|\phi^{(k+1)}-\phi^{(k)}\right\|^{2} .
$$

Proof. From convexity of $f_{2}(\phi)$ we have that,

$$
f_{2}\left(\phi^{(k+1)}\right) \geq f_{2}\left(\phi^{(k)}\right)+\left\langle\partial f_{2}\left(\phi^{(k)}\right), \phi^{(k+1)}-\phi^{(k)}\right\rangle,
$$

where, $\partial f_{2}(\phi)$ is a subgradient of $f_{2}(\phi)$. Note that the function $f_{1}(\phi)=\sum_{i=1}^{p} f_{1}\left(\phi_{i}\right)$ is separable across the coordinates and each $\phi_{i} \mapsto f_{1}\left(\phi_{i}\right)$ is strongly convex on the interval $\left(0, \frac{1}{\epsilon}\right]$ with coefficient of strong convexity: $\min _{\phi \in\left(0, \frac{1}{\epsilon}\right]} \frac{1}{\phi^{2}}=\epsilon^{2}$. If we denote $\rho\left(\geq \epsilon^{2}\right)$ as the coefficient of strong convexity for the function $f_{1}(\phi)$ on $\left(0, \frac{1}{\epsilon}\right]^{p}$ then:

$$
f_{1}\left(\phi^{(k)}\right) \geq f_{1}\left(\phi^{(k+1)}\right)+\left\langle\phi^{(k)}-\phi^{(k+1)}, \nabla f_{1}\left(\phi^{(k+1)}\right)\right\rangle+\frac{\rho}{2}\left\|\phi^{(k+1)}-\phi^{(k)}\right\|^{2},
$$

where, $\nabla f_{1}\left(\phi^{(k+1)}\right)$ is the derivative of $f_{1}(\phi)$. By standard optimality conditions [9] of Problem (36) we have:

$$
\min \left\{\left\langle\nabla F\left(\phi^{(k+1)} ; \boldsymbol{\phi}^{(k)}\right), \boldsymbol{\phi}-\boldsymbol{\phi}^{(k+1)}\right\rangle: \frac{1}{\epsilon} \mathbf{1} \geq \boldsymbol{\phi}>\mathbf{0}\right\} \geq 0
$$

where, $\nabla F\left(\phi^{(k+1)} ; \phi^{(k)}\right)=\nabla f_{1}\left(\phi^{(k+1)}\right)-\partial f_{2}\left(\phi^{(k)}\right)$ is the derivative of $\phi \mapsto F\left(\phi ; \phi^{(k)}\right)$ evaluated at $\phi^{(k+1)}$. Adding (41) and (40), and rearranging terms we get:

$$
\begin{aligned}
f_{1}\left(\phi^{(k+1)}\right)-f_{2}\left(\phi^{(k+1)}\right) \leq & f_{1}\left(\phi^{(k)}\right)-f_{2}\left(\phi^{(k)}\right)-\frac{\rho}{2}\left\|\phi^{(k+1)}-\phi^{(k)}\right\|^{2} \\
& +\underbrace{\left\langle\phi^{(k+1)}-\phi^{(k)}, \nabla f_{1}\left(\phi^{(k+1)}\right)-\partial f_{2}\left(\phi^{(k)}\right)\right\rangle}_{\leq 0} \\
& \leq f_{1}\left(\phi^{(k)}\right)-f_{2}\left(\phi^{(k)}\right)-\frac{\rho}{2}\left\|\phi^{(k+1)}-\phi^{(k)}\right\|^{2} .
\end{aligned}
$$

Where, the last line follows from 42 by setting $\phi=\phi^{(k)}$ in 42 . 
The above proposition says that $f\left(\phi^{(k)}\right)$ is a decreasing sequence; and being bounded below, converges to $\hat{f}$, say. By the definition of a first order stationary point (37), the quantity $\| \phi^{(k+1)}-$ $\phi^{(k)} \|_{2}$ dictates the proximity of $\phi^{(k)}$ to a first order stationary point; and an approximate first order stationary point. We have the following proposition, formalizing the rate at which the sequence $\phi^{(k)}$ approaches a first order stationary point.

Proposition 8. The sequence $f\left(\phi^{(k)}\right)$ is decreasing and converges to $\hat{f}$. The finite time convergence rate is given by:

$$
\min _{1 \leq k \leq \mathcal{K}} \rho\left\|\phi^{(k+1)}-\phi^{(k)}\right\|_{2}^{2} \leq \frac{2}{\mathcal{K}}\left(f\left(\phi^{(1)}\right)-\hat{f}\right) .
$$

Proof. The proof uses 39 . If $\Delta_{k}:=\frac{\rho}{2}\left\|\phi^{(k+1)}-\phi^{(k)}\right\|^{2}$ then:

$$
\mathcal{K} \min _{1 \leq k \leq \mathcal{K}} \Delta_{k} \leq \sum_{i=1}^{\mathcal{K}} \Delta_{k} \leq \sum_{i=1}^{\mathcal{K}}\left\{f\left(\phi^{(k)}\right)-f\left(\phi^{(k+1)}\right)\right\} \leq f\left(\phi^{(1)}\right)-\hat{f},
$$

where, the second inequality uses (39); and the final inequality used the fact that $f\left(\phi^{(k)}\right) \downarrow \hat{f}$. The result (43) follows from combining the left and right parts of the inequality (44).

The above proposition states that for any tolerance $\delta>0$, there is an integer $\mathcal{K}=O\left(\frac{1}{\delta}\right)$ such that for some $k \in[\mathcal{K}]$, the following holds: $\left\|\phi^{(k+1)}-\phi^{(k)}\right\|^{2} \leq \delta$.

The following proposition shows that all limit points of the sequence $\phi^{(k)}$ are first order stationary points.

Proposition 9. Any limit point of the sequence $\phi^{(k)}$ is a first order stationary point for Problem (34).

Proof. The proof is deferred to Appendix A.1

\subsection{Computational Cost}

In this section, we discuss the computational cost of Algorithm 1 and techniques for computational scalability to large problems.

When $n>p$ : The main computational cost of Algorithm 1 stems from computing a subgradient of $f_{2}(\phi)$ which requires a low rank eigen decomposition of $\mathbf{S}^{*}$. When $p$ is small relative to $n$ it is convenient to form and work with $\mathbf{S}^{*}$ (a $p \times p$ matrix). Creating $\mathbf{S}$ from $\mathbf{X}$ costs $O\left(n p^{2}\right)$ (this operation can be done once offline). Computing $\mathbf{S}^{*}$ from $\mathbf{S} \operatorname{costs} O\left(p^{2}\right)$. A full eigendecomposition of the $p \times p$ matrix $\mathbf{S}^{*}$ using dense matrix factorization methods [15] costs $O\left(p^{3}\right)$ - this approach can be used to handle problems with $p$ up to a few thousand $(p \approx 3,000$, for example). Note that this cost is of the same order as obtaining the unrestricted MLE of $\boldsymbol{\Sigma}^{-1}$ given by $\mathbf{S}^{-1}$ (assuming $\mathbf{S}$ is invertible).

When $p \gg n$ : In several applications of interest, $n$ is much smaller compared to $p$ - a situation that occurs commonly in the modern high dimensional regime (in microarray data analysis applications for example, $n$ is at most a few hundred and $p$ is in the order of tens of thousands). In such cases, obtaining a full eigen decomposition of $\mathbf{S}^{*}$ will cost $O\left(n^{2} p\right)$, which is linear in $p$ if $n \ll p$. This follows by observing that $\mathbf{S}=\frac{1}{n} \mathbf{X}^{\top} \mathbf{X}$ (where, $\mathbf{X}$ is mean-centered) and an eigen decomposition of $\mathbf{S}^{*}$, i.e., $\boldsymbol{\Phi}^{\frac{1}{2}} \mathbf{S} \boldsymbol{\Phi}^{\frac{1}{2}}=\left(\frac{1}{\sqrt{n}} \mathbf{X} \boldsymbol{\Phi}^{\frac{1}{2}}\right)^{\top}\left(\frac{1}{\sqrt{n}} \mathbf{X} \boldsymbol{\Phi}^{\frac{1}{2}}\right)$ can be obtained via a SVD of the $n \times p$ matrix $\frac{1}{\sqrt{n}} \mathbf{X} \boldsymbol{\Phi}^{\frac{1}{2}}$ - and this costs $O\left(n^{2} p\right)$. Note that this cost is significantly smaller than a direct eigendecomposition on the $p \times p$ matrix $\mathbf{S}^{*}$, which is prohibitively expensive for large values of $p$. 
In addition, there are certain costs associated with matrix multiplications. Indeed, a careful book-keeping allows us to operate with matrices that are low rank - we never need to create or form a dense $p \times p$ matrix - this is beneficial from a memory standpoint. First of all, note that the computation of $\mathbf{X} \Phi^{\frac{1}{2}}$ costs $O(n p)$. In addition, one needs to compute the diagonal entries of $T:=\boldsymbol{\Phi}^{-\frac{1}{2}} \mathbf{U D}_{1} \mathbf{U}^{\top} \boldsymbol{\Phi}^{\frac{1}{2}} \mathbf{S}$ as in $(33)$. Note that $T$ is a $p \times p$ matrix; however, its diagonal entries can be computed without explicitly creating the matrix $T$. This follows by observing that $T$ is the outer product of two low rank matrices: $T:=T_{1} T_{2}$, where, $T_{1}=\boldsymbol{\Phi}^{-\frac{1}{2}} \mathbf{U D}_{1}$ and $T_{2}=\mathbf{U}^{\top} \boldsymbol{\Phi}^{\frac{1}{2}} \mathbf{S}$ - here, $T_{1} \in \Re^{p \times r}$ (recall that $r<n$ ); and $T_{2} \in \Re^{r \times p}$. Computing the diagonal entries of $T$ has a cost $O(p r)$. Note that $T_{2}$ has to be computed carefully using transitivity, by observing that $T_{2}=T_{21} \mathbf{X}$ with $T_{21}=\frac{1}{n} \mathbf{U}^{\top}\left(\boldsymbol{\Phi}^{\frac{1}{2}} \mathbf{X}^{\prime}\right) \in \Re^{r \times n}$ — thus we can avoid creating/storing a matrix of size $p \times p$.

When both $p, n$ are large: When both $n, p$ are large, direct SVD factorization methods above become computationally expensive. Approximate schemes for large scale low rank SVD decompositions are called for. Approximate rank $r$ eigen decompositions of $\mathbf{S}^{*}$ can be computed using incremental eigendecomposition techniques as described in [11]; or methods based on the Lanczos method [18] or block power iterations [15]. All these methods will cost roughly $O\left(p^{2} r\right)$ and can be significantly smaller (both in terms of memory and computational cost) when compared to a full eigendecomposition with cost $O\left(p^{3}\right)$ for $r \ll p$.

\subsection{Solutions to Problem (2) when $\epsilon \approx 0$}

The conventional version of the ML factor analysis optimization problem is given by:

$$
\begin{aligned}
\operatorname{minimize} & -\log \operatorname{det}\left(\boldsymbol{\Sigma}^{-1}\right)+\operatorname{tr}\left(\boldsymbol{\Sigma}^{-1} \mathbf{S}\right) \\
\text { s.t. } & \boldsymbol{\Sigma}=\boldsymbol{\Psi}+\mathbf{L} \mathbf{L}^{\top}, \boldsymbol{\Psi}=\operatorname{diag}\left(\psi_{1}, \ldots, \psi_{p}\right) \succ \mathbf{0},
\end{aligned}
$$

which may be interpreted as a limiting version of Problem (2) with $\epsilon \rightarrow 0+$. There are certain technical difficulties with Problem (45) as it may be unbounded below and hence a ML estimator need not exist. This can lead to numerically unstable algorithms which are highly undesirable from a practical standpoint. 28 discuss necessary and sufficient condition for boundedness of Problem (45). Problem (45) is bounded below under the following conditions: (a) If $\mathbf{S}$ is of full rank or (b) If $\mathbf{S}$ is rank deficient, then $r<s-1$ where, $s$ denotes the number of nonzero coordinates in the sparsest nonzero vector (i.e., a nonzero vector with the maximal number of zero coefficients) in the null space of $\mathbf{S}$. Computing the sparsest nonzero vector in a subspace, however, is a combinatorially difficult problem. Note that if the sample covariance matrix corresponds to that of a continuous random variable, then Problem (45) is bounded below with probability one. Even if Problem 45 is bounded below, the minimum may not be attained - the infimum is attained when some coordinates of $\boldsymbol{\Psi}$ are set to zero. These are known as Heywood cases [28] - and they are well-known to create numerical difficulties from a computational viewpoint and also misleading inference from a statistical viewpoint.

If $\hat{\boldsymbol{\Psi}}_{\epsilon}$ is a solution to Problem (2); then the limiting value of $\hat{\boldsymbol{\Psi}}_{\epsilon}$ as $\epsilon \downarrow 0+$ will give a solution to Problem (45), provided it exists. This inspires a simple continuation scheme by considering a sequence of $\epsilon$-values converging to a small number $\epsilon \downarrow \epsilon^{\prime}$ (with $\epsilon^{\prime}:=10^{-6}$, say); and using warm-starts for Algorithm 1. Suppose, there is a subset of indices $\mathbf{M} \subset\{1, \ldots, p\}$ such that, $\hat{\psi}_{i}=\epsilon^{\prime}$ for all $i \in \mathbf{M}$, and $\hat{\psi}_{i}>\epsilon^{\prime}$ for all $i \notin \mathbf{M}$, then we obtain an upper bound to Problem (45) by fixing $\psi_{i}=\epsilon^{\prime}$ for all $i \in \mathbf{M}$ and optimizing over the remaining $\psi_{i}, i \notin \mathbf{M}$ values. This can be performed by a simple modification to Algorithm 1, in the update (36), where, we update only the $\phi_{i}$ values (recall that $\phi_{i}=\psi_{i}^{-1}$ for all $i$ ) corresponding to $i \notin \mathbf{M}$ and set the remaining $\phi_{i}$ values to $1 / \epsilon^{\prime}$. 


\subsection{Ridge Regularization}

Instead of considering a direct lower bound on $\psi_{i}$ 's as in Problem (2), we can also consider a ridge regularized version of Problem (45) given by:

$$
\text { minimize } f(\phi)+\sum_{i=1}^{p} \gamma \phi_{i}^{2} \quad \text { s.t. } \quad \boldsymbol{\phi}=\operatorname{diag}\left(\phi_{1}, \ldots, \phi_{p}\right) \succ \mathbf{0},
$$

for some $\gamma>0$. Algorithm 1 can be adapted to Problem (46) by changing update (36) as:

$$
\phi^{(k+1)}=\underset{\phi_{i}>0, \forall i}{\arg \min } \sum_{i \in[p]}\left\{-\log \phi_{i}+s_{i i} \phi_{i}+\gamma \phi_{i}^{2}-\nabla_{k, i} \phi_{i}\right\}
$$

where, $\nabla_{k, i}$ 's can be computed as in Section 2.2.1. The $i$ th coordinate of $\phi^{(k+1)}$ is given by:

$$
\phi_{i}^{(k+1)}=\frac{1}{4 \gamma}\left(\nabla_{k, i}-s_{i i}+\sqrt{\left(s_{i i}-\nabla_{k, i}\right)^{2}+8 \gamma}\right), \quad 1 \leq i \leq p .
$$

Note that $s_{i i}-\nabla_{k, i}$ is the $i$ th diagonal of the matrix $\mathbf{S}-\boldsymbol{\Phi}^{-\frac{1}{2}} \mathbf{U} \mathbf{D}_{1} \mathbf{U}^{\top} \boldsymbol{\Phi}^{\frac{1}{2}} \mathbf{S}$, which can be rearranged as:

$$
\boldsymbol{\Phi}^{-\frac{1}{2}}\left(\boldsymbol{\Phi}^{\frac{1}{2}} \mathbf{S} \boldsymbol{\Phi}^{\frac{1}{2}}-\mathbf{U D}_{1} \mathbf{U}^{\top} \boldsymbol{\Phi}^{\frac{1}{2}} \mathbf{S} \boldsymbol{\Phi}^{\frac{1}{2}}\right) \boldsymbol{\Phi}^{-\frac{1}{2}}=\boldsymbol{\Phi}^{-\frac{1}{2}}\left(\mathbf{U} \widetilde{\mathbf{D}} \mathbf{U}^{\top}\right) \boldsymbol{\Phi}^{-\frac{1}{2}}
$$

where $\widetilde{\mathbf{D}}$ is a diagonal matrix with diagonal entries given by

$$
\widetilde{d}_{i i}= \begin{cases}1 & \text { if } 1 \leq i \leq r \text { and } \lambda_{i}^{*} \geq 1 \\ \lambda_{i}^{*} & \text { otherwise }\end{cases}
$$

This implies that the matrix in 49 is positive semidefinite; and in particular, the diagonal entries are nonnegative: $s_{i i}-\nabla_{k, i} \geq 0$ for all $i \in[p]$. This implies from (48) that: $\psi_{i}^{(k+1)}=$ $\frac{1}{\phi_{i}^{(k+1)}} \geq \sqrt{2 \gamma}$. Hence ridge regularization keeps the estimated $\psi_{i}$ values bounded away from zero. A continuation scheme similar to that described in Section 2.6 can be used with $\gamma \downarrow \gamma^{\prime}$ (with $\gamma^{\prime}=10^{-8}$, say) to get a good solution to Problem 45).

\section{Computational Experiments}

We present computational experiments on our proposed Algorithm 1 (aka FACTMLE). We compare its performance versus other popular approaches for ML factor analysis on synthetic and real-data examples. All computations were done in Matlab on a Mac desktop with 32 GB RAM.

\subsection{Comparison across different methods}

In this section we compare the performance of the method proposed herein: FACTMLE with leading algorithms for ML factor analysis: Fa, factoran and Emfact.

Synthetic data generation: We generated (the true) $\mathbf{L}^{0} \in \Re^{p \times r_{0}}$ (with $r_{0} \ll p$ ) with entries drawn iid from $N\left(\mu, \sigma^{2}\right)$. For examples with $n>p$, shown in Figure 1 , we set $\mu=10$ and $\sigma^{2}=1$. The (true) unique variances $\boldsymbol{\Psi}^{0}=\operatorname{diag}\left(\psi_{1}^{0}, \psi_{2}^{0}, \ldots, \psi_{p}^{0}\right)$ were generated independently from an exponential distribution with mean 10. Once $\boldsymbol{\Psi}^{0}$ and $\mathbf{L}^{0}$ were generated, we created $\boldsymbol{\Sigma}^{0}=\boldsymbol{\Psi}^{0}+\mathbf{L}^{0} \mathbf{L}^{0^{\top}}$. We generated $\mathbf{X}_{n \times p}$ from a multivariate Gaussian distribution with mean $\mathbf{0}$ and covariance $\boldsymbol{\Sigma}$. The results were averaged over ten different replications of $\mathbf{X}$.

Competing methods: We compared our proposed method: Algorithm 1 (FACTMLE) with the following popular ML methods for Factor Analysis: 
1. Factoran: This is the widely used, native implementation of ML factor analysis in Matlab; and this code is based on the seminal work of [17.

2. Emfact: This is the widely popular EM algorithm based technique; and the specific implementation is based on [3].

3. Fa: This is an EM algorithm based technique for factor analysis 8

Of the above three methods, Factoran and Emfact apply only when $n>p$ - thus we restrict our attention to $\mathrm{Fa}$ as the only competing method to FACTMLE for $n<p$. We do not include the method of [7] in our comparisons, since it optimizes a different criterion (not the maximum likelihood objective). In terms of scalability considerations, the method of [7] is less scalable (since it requires performing the eigen decomposition of an unstructured $p \times p$ matrix) than FACTMLE especially if $n$ is small. The standard implementation of Factoran uses correlation matrices; hence we transformed the results obtained from Fcatoran for comparison with other algorithms.

Performance measures: All algorithms are compared in terms of the quality of solution obtained, i.e., the objective value - the criteria they are all set to optimize. We consider the negative $\log$-likelihood $\mathcal{L}(\hat{\boldsymbol{\Sigma}}):=-\log \operatorname{det}\left(\hat{\boldsymbol{\Sigma}}^{-1}\right)+\operatorname{tr}\left(\hat{\boldsymbol{\Sigma}}^{-1} \mathbf{S}\right)$, where, $\hat{\boldsymbol{\Sigma}}=\hat{\boldsymbol{\Psi}}+\hat{\mathbf{L}}^{\top} \hat{\mathbf{L}}^{\top}$, upon verifying that the estimates are feasible.

In addition, we also consider the run-times of the different algorithms. This however, depends upon the different convergence criteria employed by the different algorithms (as we explain below); and the quality of solution obtained. We note that the quality of solution (in terms of objective value) can be different for different algorithms since the optimization problem is nonconvex. Thus run-time of an algorithm is interpreted in the context of the objective value attained in our experiments.

Finally, we note that Algorithm 1 can readily incorporate warm-starts - they may be useful if one desires a sequence of solutions to Problem (2) for different values of $r=1,2, \ldots$. Other algorithms like: Fa, Factoran and Emfact do not allow for warm-start specification; and we used their default initialization strategy.

Comparison results: We first consider a synthetic dataset with $n>p$. Figure 1 shows the performances of different methods for synthetic data generated as above, with $p=200, n=$ $2200, r^{0}=8$, and different choices of $r$. We compare the performance of the different algorithms in terms of their default convergence criteria.

For FACTMLE, the tolerance level $\eta$ (for convergence based on objective value difference, as explained in Section 2.3 was set to $10^{-4}$; we set the maximum number of iterations to be 1000 . For all other we choose their default convergence criteria with maximum number of iterations set to 1000. Figure 1 suggests that the performance of FACTMLE measured in terms of the negative log-likelihood is significantly better than all the other algorithms - thereby suggesting that it does well in the task it was set to accomplish. The performance of Emfact, in terms of negative log-likelihood, is comparable to FACTMLE when $r$ is small. However, Emfact often encounters numerical difficulties (especially when $r$ is large) and produces negative estimates of $\boldsymbol{\Phi}$ - this violates the condition $\mathbf{\Phi} \succ \mathbf{0}$ and is highly undesirable from a statistical standpoint. Consequently, one should be cautious while using this method. A highly attractive feature of FACTMLE is the timings. The number of iterations required in Fa, Emfact and Factoran is much larger than that of FACTMLE (note that in addition, their objective values are worse as well). For example, for $p=200, r=6$ the (average) number of iterations for Fa, Emf act,Factoran were around 1000 (i.e., the maximal iteration limit), but for FACTMLE it was less than twenty. We observe that the difference among FACTMLE and its competitors is more pronounced for larger values of $r$. In terms of timings, FACTMLE is a clear winner - and the differences are more

${ }^{5}$ This function is available as a part of Matlab's PRML toolbox https://www.mathworks.com/matlabcentral/ fileexchange/55883-probabilistic-pca-and-factor-analysis?focused=6047050\&tab=function 
pronounced with larger values of $p$ (as we see in subsequent experiments). The performance $\mathrm{Fa}$ is better than Factoran and Emfact; and seems to be the only competitor to FACTMLE. We perform a more detailed experiment comparing the performance of Fa with Algorithm 1 for two different types of initializations: with warm start and with random initialization in Figure 1 (bottom panel). We see that in both cases FACTMLE outperforms Fa in terms of quality of solution and also run-times. The timings of FACTMLE with warm start is found to be slightly better than FACTMLE with cold start.

We also took some other values of $(n, p, r)$ but the results were found to be quite similar; and hence we do not report them here.
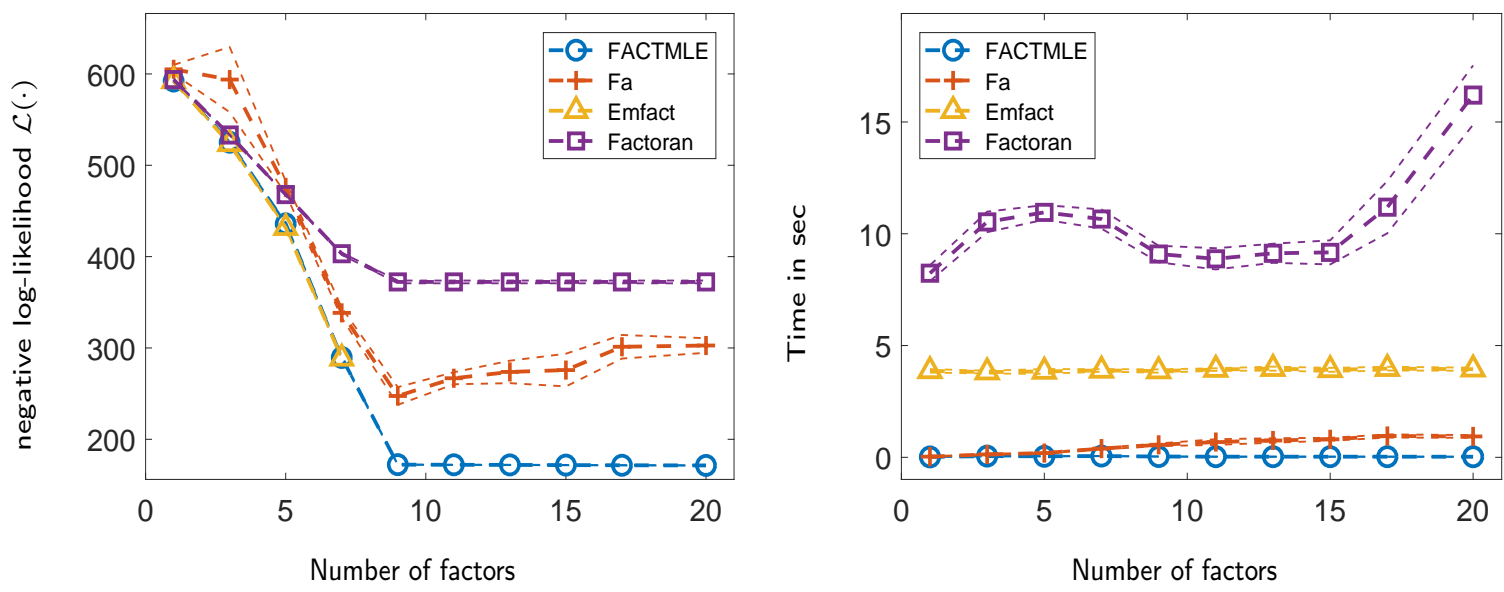

Zoomed Plot: FACTMLE vs Fa
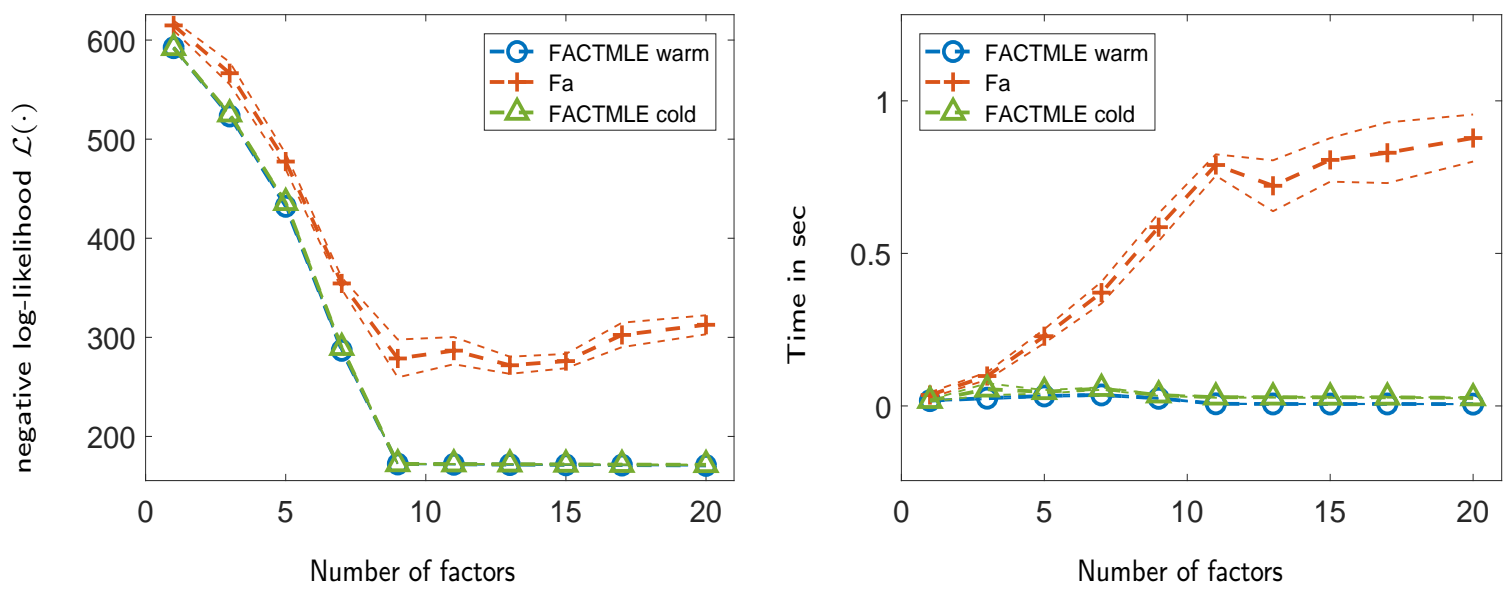

Figure 1: Figure showing performances of different ML methods for Factor Analysis for synthetic data with $(n, p)=(2200,200)$. In the two figures in first row, we compare the performance of Fa, FACTMLE, Factoran and Emfact in terms of negative log-likelihood and run-time. We see that our method Factmle outperforms all other methods, both in terms of run-time and negative log-likelihood value. Emfact produces negative estimates of $\boldsymbol{\Phi}$ in some cases, and in those cases the result obtained from Emfact do not appear in the plots. The lower panel shows a zoomed-in version of $\mathrm{Fa}$ (the best competitor of FACTMLE) versus FACTMLE with warm start and without warm-start (denoted as "cold"). We see that the performance of FACTMLE (with or without warm start) is better than that of Fa. FACTMLE with warm start gives slightly better run-time. Results are averaged over ten replications and the bands represent point-wise one standard error bars. 


\subsubsection{Further comparisons with $\mathrm{Fa}$}

In our experience, we systematically observed that among Fa, Factoran and Emfact; Fa always emerged as the winner (see also Figure 1) in terms of numerical stability, quality of solutions delivered and run-times. We also note that $\mathrm{Fa}$ is the only method among the three which applies for both $n>p$ and $n \leq p$. Hence we perform a more detailed comparison between Fa and our proposed method: FACTMLE. We consider four different datasets in our experiments one synthetic and the other real - this includes both the cases $n>p$ and $p>n$. For all the numerical examples in this section, we had the same convergence criterion for both Fa and FACTMLE. We ran both Fa and FACTMLE for a maximum of 2000 iterations and tolerance threshold of $\eta=10^{-8}$ (as described in the display of Algorithm 1). If $f_{k}(\mathrm{alg})$ denotes the objective value (negative $\log$-likelihood) for method alg $\in\{\mathrm{Fa}, \mathrm{FACTMLE}\}$ at iteration $k$; then we obtain the best objective value across all the methods and set it to $f_{*}$. We then study the first time at which an algorithm "alg" reaches a tolerance level of "Tol": $\left(f_{k}(\operatorname{alg})-f_{*}\right) /\left|f_{*}\right| \leq$ Tol.

We considered the following datasets in our experiments.

Example a: (Simulated Data) $(p>n)$ : We consider 3 synthetic datasets where, the number of covariates $p$ is larger than the number of samples $n$; we took: $(n, p)=(500,5000)$, $(n, p)=\left(50,10^{4}\right)$ and $(n, p)=\left(150,10^{4}\right)$. The data was simulated as per the setup mentioned in Section 3.1, with the entries $\phi_{i}^{0}$ drawn iid from an exponential distribution with mean 1 . We set $r^{0}=5$. We considered a sequence of solutions for the ML factor analysis problem for 15 equi-spaced values of $r \in[1,18]$.

Example b: (Phoneme Data), $(n>p)$ : The data were extracted from the TIMIT database a widely used resource for research in speech recognition. The data was downloaded from the companion website ${ }^{6}$ of the textbook [34 - it consists of 4509 log-periodograms of length 256, i.e. in this example $(n, p)=(4509,256)$. We considered a sequence of solutions for the ML factor analysis problem for 18 equi-spaced values of $r \in[1,27]$. The sample covariance matrix $\mathbf{S}$ was poorly conditioned. The condition number of $\mathbf{S}$ was $\sim 3.9 \times 10^{3}$.

Example c: (ZipCode Data), $(n>p)$ : This is the by now famous ZipCode dataset 7 which was generated by scanning normalized handwritten digits by the U.S. Postal Service. These are $16 \times 16$ grayscale images corresponding to digits (0-9) that are normalized/deslanted. The images were vectorized, and we created a data matrix comprising of digits 0 and 6 . The data matrix $\mathbf{X}_{n \times p}$ had dimensions $(n, p)=(1858,249)$. We considered a sequence of solutions for the ML factor analysis problem for 15 equi-spaced values of $r \in[1,17]$. The condition number of $\mathbf{S}$ here was $\sim 3.8 \times 10^{17}$.

Example d: (Cancer Data), $(p>n)$ : This is a high-dimensional microarray dataset available from ${ }^{8}$ the companion website of 34 . It consists of gene expression measurements for $p=16063$ genes from $n=144$ individuals. We considered a sequence of solutions for the ML factor analysis problem for 15 equi-spaced values of $r \in[1,22]$. The condition number of $\mathbf{S}$ here was $\sim 10^{20}$.

For Examples b,c,d, the condition number of $\mathbf{S}$ was quite high — we took $\epsilon=10^{-10}$ (in Problem (2) and Algorithm 1 was provided with this choice of $\epsilon$. For Algorithm 1, we did not use the continuation strategy as described in Section 2.6. For the synthetic dataset (Example a), we took $\epsilon=10^{-3}$. In Algorithm 1, the diagonal entries of $\boldsymbol{\Phi}$ were drawn from a uniform $[0,1]$ distribution for the case $r=1$; and solutions for other values of $r$ were obtained via warm-starts. For $\mathrm{Fa}$ we used its default initialization scheme since it does not allow for warm-starts.

For all cases with $n<p$, the low rank SVD step in Algorithm 1 was performed according to the description given in Section 2.5 (for the case $p \gg n$ ). For $n>p$, we used the low rank SVD method of Section 2.5 (for the case $n>p$ ).

\footnotetext{
${ }^{6}$ Available at: https://web.stanford.edu/ hastie/ElemStatLearn/datasets/phoneme.data

${ }^{7}$ Available at https://web.stanford.edu/ ${ }^{\sim}$ hastie/ElemStatLearn/datasets/zip.test.gz

${ }^{8}$ Data available at http://statweb.stanford.edu/ tibs/ElemStatLearn/datasets/14cancer.info
} 
Table 1 shows the results for all the four examples. We observe that FACTMLE clearly works extremely well in terms of obtaining a good objective value in much smaller run-times, compared to Fa. What is most important however, is that FACTMLE is numerically robust - in fact much more stable and reliable than $\mathrm{Fa}$ which often encounters problems with convergence. In many cases, as soon as $r$ is not too small, Fa is found to be trapped in poor fixed points with suboptimal objective values. These are referred to by the moniker " $>$ *" in Table 1. We note that these datasets are challenging as the condition numbers associated with them are extremely large however, we do expect to encounter datasets of this form often in real-life settings, thus having a good robust algorithm is of paramount importance. Our proposed algorithm FACTMLE does seem to meet this expectation.

Synthetic Data $(n=500, p=5000)$

Time (in secs)

\begin{tabular}{|c|c|c|}
\hline Tol & Fa & FACTMLE \\
\hline $10^{-2}$ & $>^{*} 83.142(4.174)$ & $4.497(0.041)$ \\
\hline $10^{-3}$ & $>^{*} 107.713(2.148)$ & $7.702(2.508)$ \\
\hline $10^{-4}$ & $>^{*} 112.145(1.157)$ & $18.490(5.706)$ \\
\hline $10^{-5}$ & $>^{*} 112.146(1.157)$ & $29.369(6.154)$ \\
\hline
\end{tabular}

Synthetic Data $\left(n=50, p=10^{4}\right)$

Time (in secs)

\begin{tabular}{|c|cc|cc|}
\hline Tol & \multicolumn{2}{|c|}{ Fa } & \multicolumn{2}{c|}{ FACTMLE } \\
\hline $10^{-2}$ & $>^{*} 43.794$ & $(1.160)$ & 0.558 & $(0.097)$ \\
\hline $10^{-3}$ & $>^{*} 50.798$ & $(0.712)$ & 0.882 & $(0.228)$ \\
\hline $10^{-4}$ & $>^{*} 52.669$ & $(0.362)$ & 1.901 & $(0.375)$ \\
\hline $10^{-5}$ & $>^{*} 52.959$ & $(0.296)$ & 3.798 & $(0.496)$ \\
\hline
\end{tabular}

ZipCode Data $(n, p)=(1858,249)$ Time (in secs)

\begin{tabular}{|c|cc|cc|}
\hline Tol & \multicolumn{2}{|c|}{ Fa } & \multicolumn{2}{c|}{ FACTMLE } \\
\hline $10^{-2}$ & \multicolumn{2}{|c|}{$1.558(0.060)$} & 0.111 & $(0.007)$ \\
\hline $10^{-3}$ & \multicolumn{2}{|c|}{$5.822(0.099)$} & 0.171 & $(0.008)$ \\
\hline $10^{-4}$ & $>^{*} 8.673(0.100)$ & 0.445 & $(0.010)$ \\
\hline $10^{-5}$ & $>^{*} 10.803(0.099)$ & 0.640 & $(0.012)$ \\
\hline
\end{tabular}

Synthetic Data $\left(n=150, p=10^{4}\right)$ Time (in secs)

\begin{tabular}{|c|c|c|}
\hline Tol & Fa & FACTMLE \\
\hline $10^{-2}$ & $>^{*} 48.489(4.605)$ & $1.680(0.027)$ \\
\hline $10^{-3}$ & $>^{*} 90.445(1.601)$ & $1.920(0.048)$ \\
\hline $10^{-4}$ & $>^{*} 93.004(0.774)$ & $4.393(0.592)$ \\
\hline $10^{-5}$ & $>^{*} 93.004(0.773)$ & $6.662(0.764)$ \\
\hline
\end{tabular}

Phoneme Data $(n, p)=(4509,256)$

Time (in secs)

\begin{tabular}{|c|cc|cc|}
\hline Tol & \multicolumn{2}{|c|}{ Fa } & \multicolumn{2}{c|}{ FACTMLE } \\
\hline $10^{-2}$ & \multicolumn{2}{|c|}{$3.038(0.385)$} & 0.125 & $(0.006)$ \\
\hline $10^{-3}$ & $>^{*} 23.902(1.624)$ & 0.187 & $(0.007)$ \\
\hline $10^{-4}$ & $>^{*} 29.635(1.430)$ & 0.420 & $(0.007)$ \\
\hline $10^{-5}$ & $>^{*} 37.035(1.245)$ & 0.669 & $(0.008)$ \\
\hline
\end{tabular}

Cancer Data $(n=144, p=16063)$

Time (in secs)

\begin{tabular}{|c|c|c|}
\hline Tol & $\mathrm{Fa}$ & FACTMLE \\
\hline $10^{-2}$ & $1.055(0.016)$ & $3.816 \quad(0.014)$ \\
\hline $10^{-3}$ & $12.021 \quad(0.099)$ & $\begin{array}{ll}5.346 & (0.040)\end{array}$ \\
\hline $10^{-4}$ & $>^{*} 81.979 \quad(0.355)$ & $8.516(0.050)$ \\
\hline $10^{-5}$ & $>^{*} 94.586 \quad(0.357)$ & $12.431 \quad(0.060)$ \\
\hline
\end{tabular}

Table 1: Performance of FACTMLE and Fa for different real and synthetic datasets; as described in the text. We show the times (secs) taken by different algorithms to compute the entire path of solutions for different values of $r$, as specified in the text. In all the above examples, a symbol " $>$ *" means that the corresponding algorithm did not converge to the specified tolerance level for multiple values of $r$ and replications. This usually does not happen when $r$ is small (say less than 5), but is indeed a common problem whenever $r$ becomes larger. FACTMLE is seen to be a clear winner across all instances. The results are averaged over 10 replications with standard errors in parenthesis.

\section{Generalizing beyond a diagonal $\Phi$}

The most conventional version of FA concerns a diagonal matrix and the focus of the paper is on this case. However, we show that our proposed framework can also naturally adapt to more 
general constraints on $\boldsymbol{\Phi}$. Towards this end, we have the following remark:

Remark 2. For any $r \in[p]$, it follows from the proof of Proposition 5 , that for any $\mathbf{\Phi} \succeq \mathbf{0}$ (not necessarily diagonal) the function

$$
\mathbf{\Phi} \mapsto F_{2}(\boldsymbol{\Phi}):=\sum_{i=1}^{r}\left(\log \left(\max \left\{1, \lambda_{i}^{*}\right\}\right)-\max \left\{1, \lambda_{i}^{*}\right\}+1\right)
$$

where, $\left\{\lambda_{i}^{*}\right\}_{1}^{p}$ are the eigenvalues of $\mathbf{S}^{\frac{1}{2}} \mathbf{\Phi} \mathbf{S}^{\frac{1}{2}}$ is concave in $\mathbf{\Phi} \succeq \mathbf{0}$.

For a general $\boldsymbol{\Psi} \succ \mathbf{0}$, the maximum likelihood FA problem:

$$
\text { minimize }-\log \operatorname{det}\left(\boldsymbol{\Sigma}^{-1}\right)+\operatorname{tr}\left(\boldsymbol{\Sigma}^{-1} \mathbf{S}\right) \quad \text { s.t. } \boldsymbol{\Sigma}=\boldsymbol{\Psi}+\mathbf{L} \mathbf{L}^{\top}, \boldsymbol{\Psi} \succ \mathbf{0},
$$

with optimization variables $\boldsymbol{\Sigma}, \mathbf{L}, \boldsymbol{\Psi}$ (with same dimensions as before) is equivalent to

$$
\text { minimize } \quad H(\boldsymbol{\Phi}):=F_{1}(\boldsymbol{\Phi})-F_{2}(\boldsymbol{\Phi}) \quad \text { s.t. } \quad \boldsymbol{\Phi} \succ \mathbf{0},
$$

where, $F_{1}(\boldsymbol{\Phi}):=-\log \operatorname{det}(\boldsymbol{\Phi})+\operatorname{tr}(\boldsymbol{\Phi} \mathbf{S})$; and $F_{2}(\boldsymbol{\Phi})$ is as defined above (Remark 2). Note that $F_{1}(\boldsymbol{\Phi})$ and $F_{2}(\boldsymbol{\Phi})$ are convex in $\boldsymbol{\Phi}$. However, in the context of FA it is meaningful to consider additional regularization on $\boldsymbol{\Phi}$. For example, $\boldsymbol{\Phi}$ can be block-diagonal; banded or have entries with small $\ell_{1}$-norm (aka $\ell_{1}$-sparsity).

In presence of additional convex constraints on $\boldsymbol{\Phi}$, i.e., say, $\boldsymbol{\Phi} \in \mathrm{X}$ the difference of convex optimization procedure described in Section 2.3 readily applies. Algorithm 1 gets modified to the following convex problem with semidefinite constraints

$$
\mathbf{\Phi}^{(k+1)} \in \arg \min \left\{F_{1}(\boldsymbol{\Phi})-\left\langle\partial F_{2}\left(\boldsymbol{\Phi}^{(k)}\right), \boldsymbol{\Phi}\right\rangle: \boldsymbol{\Phi} \in \mathbf{X}, \boldsymbol{\Phi} \succ \mathbf{0}\right\} .
$$

Clearly, the efficiency of this procedure depends upon how efficiently the subproblem 52 can be solved - this depends upon X. If $\boldsymbol{\Phi}$ is banded, block diagonal, or has small $\ell_{1}$-norm, X can be described by a polyhedral set.

A special structure of $\boldsymbol{\Phi}$ is when it is block diagonal $\boldsymbol{\Phi}=\operatorname{Blkdiag}\left(\boldsymbol{\Phi}_{1}, \ldots, \boldsymbol{\Phi}_{m}\right)$ where, $\boldsymbol{\Phi}_{i} \in \Re^{p_{i} \times p_{i}}$ is the $i$ th block, for $i=1, \ldots, m$. If $\mathbf{X}=\{\boldsymbol{\Phi}: \mathbf{\Phi} \succeq \mathbf{0}\}$, then the optimization problem (52) splits into $m$ different blocks; and the solution for the $i$ th block is given by: $\boldsymbol{\Phi}_{i}^{(k+1)}=\left(\mathbf{S}_{i i}-\nabla_{k, i i}\right)^{-1}$, where, $\mathbf{S}_{i i}\left(\right.$ and $\left.\boldsymbol{\nabla}_{k, i i}\right)$ are the $i$ th blocks of $\mathbf{S}$ (respectively, $\left.\boldsymbol{\nabla}_{k}\right)$ :

$$
\mathbf{S}=\left[\begin{array}{cccc}
\mathbf{S}_{11} & \mathbf{S}_{12} & \cdots & \mathbf{S}_{1 m} \\
\vdots & & \ddots & \vdots \\
\mathbf{S}_{m 1} & \mathbf{S}_{m 2} & \cdots & \mathbf{S}_{m m}
\end{array}\right] \text { and } \boldsymbol{\nabla}_{k}=\left[\begin{array}{cccc}
\boldsymbol{\nabla}_{k, 11} & \boldsymbol{\nabla}_{k, 12} & \cdots & \boldsymbol{\nabla}_{k, 1 m} \\
\vdots & & \ddots & \vdots \\
\boldsymbol{\nabla}_{k, m 1} & \boldsymbol{\nabla}_{k, m 2} & \cdots & \boldsymbol{\nabla}_{k, m m}
\end{array}\right]
$$

where, $\mathbf{S}_{i j}$ (and $\boldsymbol{\nabla}_{i j}$ ) is $p_{i} \times p_{j}$ submatrix of $\mathbf{S}$ (respectively, $\boldsymbol{\nabla}_{k}$ ). Note that $\boldsymbol{\nabla}_{k}$ is a subgradient of $F_{2}(\boldsymbol{\Phi})$ w.r.t. $\boldsymbol{\Phi}$ evaluated at $\boldsymbol{\Phi}^{(k)}$.

One can also consider an $\ell_{1}$-norm sparsity on the entries of $\boldsymbol{\Phi}$, akin to graphical lasso [14, 5 ] commonly used in learning sparse inverse covariance matrices. In this case, update (52) leads to an optimization problem of the form:

$$
\operatorname{minimize} \quad-\log \operatorname{det}(\boldsymbol{\Phi})+\left\langle\mathbf{S}-\nabla_{k}, \boldsymbol{\Phi}\right\rangle+\lambda \sum_{i j}\left|\phi_{i j}\right| \quad \text { s.t. } \quad \boldsymbol{\Phi} \succeq \mathbf{0}, \quad \phi_{i j} \geq 0 \quad \forall i, j ;
$$

which can be solved using adaptions of standard algorithms for the graphical lasso - see [14, 34, 5 . 


\section{A Appendix}

Proposition 10. (See Section 6 in [9]) Suppose the function $g: \mathbf{E} \mapsto(-\infty, \infty)$ is convex, and the point $x$ lies in interior of $\operatorname{dom}(g)$ with $\mathbf{E} \subset \Re^{m}$. If $x^{r} \rightarrow x$ (where, $x^{r}, x \in \mathbf{E}$ ) and $\nu^{r} \rightarrow \nu$; as $r \rightarrow \infty$, where, $\nu^{r}$ is a subgradient of $g$ evaluated at $x^{r}$. Then $\nu$ is a subgradient of $g$ evaluated at $x$.

\section{A.1 Proof of Proposition 9}

Note that the objective function $f(\phi)$ (see (22)) is unbounded above when $\phi_{i} \rightarrow 0$ for any $i \in[p]$ - see also Proposition 3. This implies that there exists a $\alpha>0$ such that $\phi^{(k)} \in\left[\alpha, \frac{1}{\epsilon}\right]^{p}$ for all $k$ (sufficiently large). The boundedness of $\phi^{(k)}$ implies the existence of a limit point of $\phi^{(k)}$, say, $\phi^{*}$. Let $\phi^{\left(k_{j}\right)}$ be a subsequence such that $\phi^{\left(k_{j}\right)} \rightarrow \phi^{*}$ as $j \rightarrow \infty$. Note that for every $k$, $\phi^{(k+1)} \in \arg \min _{\boldsymbol{\phi} \in \mathcal{C}} F\left(\phi ; \phi^{(k)}\right)$ is equivalent to

$$
\left\langle\nabla f_{1}\left(\phi^{(k+1)}\right)-\partial f_{2}\left(\phi^{(k)}\right), \phi-\phi^{(k+1)}\right\rangle \geq 0 \quad \forall \phi \in \mathcal{C} .
$$

Now consider the sequence $\boldsymbol{\phi}^{\left(k_{j}\right)}$ as $j \rightarrow \infty$. Using the fact that $\boldsymbol{\phi}^{(k+1)}-\boldsymbol{\phi}^{(k)} \rightarrow \mathbf{0}$; it follows from the continuity of $\nabla f_{1}(\cdot)$ that: $\nabla f_{1}\left(\phi^{\left(k_{j}+1\right)}\right) \rightarrow \nabla f_{1}\left(\phi^{*}\right)$ as $j \rightarrow \infty$.

Note that $\partial f_{2}\left(\phi^{\left(k_{j}\right)}\right)$ (see (33)) is bounded as $\phi^{\left(k_{j}\right)} \in\left[\alpha, \frac{1}{\epsilon}\right]^{p}$. Passing onto a further subsequence $\left\{k_{j}^{\prime}\right\}$ if necessary, it follows that $\partial f_{2}\left(\phi^{\left(k_{j}^{\prime}\right)}\right) \rightarrow \vartheta$. Using Proposition 10 , we conclude that $\vartheta$ is a subgradient of $f_{2}$ evaluated at $\phi^{*}$. As $k_{j}^{\prime} \rightarrow \infty$, the above argument along with (54) implies that:

$$
\left\langle\nabla f_{1}\left(\boldsymbol{\phi}^{*}\right)-\partial f_{2}\left(\boldsymbol{\phi}^{*}\right), \boldsymbol{\phi}-\boldsymbol{\phi}^{*}\right\rangle \geq 0 \quad \forall \boldsymbol{\phi} \in \mathcal{C},
$$

where, $\partial f_{2}\left(\phi^{*}\right)$ is a subgradient of $f_{2}$ evaluated at $\phi^{*}$. (55) implies that $\phi^{*}$ is a first order stationary point.

\section{References}

[1] Ahn, M., J.-S. Pang, and J. Xin (2017). Difference-of-convex learning: directional stationarity, optimality, and sparsity. SIAM Journal on Optimization 27(3), 1637-1665.

[2] Anderson, T. (2003). An Introduction to Multivariate Statistical Analysis (3rd ed.). Wiley, New York.

[3] Bai, J. and K. Li (2012). Statistical analysis of factor models of high dimension. The Annals of Statistics 40(1), 436-465.

[4] Bai, J. and S. Ng (2008). Large dimensional factor analysis. Foundations and Trends in Econometrics 3(2), 89-163.

[5] Banerjee, O., L. E. Ghaoui, and A. d'Aspremont (2008). Model selection through sparse maximum likelihood estimation for multivariate gaussian or binary data. Journal of Machine Learning Research 9, 485-516.

[6] Bartholomew, D., M. Knott, and I. Moustaki (2011). Latent Variable Models and Factor Analysis: A Unified Approach. John Wiley and Sons, United Kingdom.

[7] Bertsimas, D., M. S. Copenhaver, and R. Mazumder (2017). Certifiably optimal low rank factor analysis. Journal of Machine Learning Research 18(29), 1-53.

[8] Bishop, C. (2006). Pattern Recognition and Machine Learning. Springer, New York.

[9] Borwein, J. and A. Lewis (2006). Convex Analysis and Nonlinear Optimization. Springer.

[10] Boyd, S. and L. Vandenberghe (2004). Convex Optimization. Cambridge University Press, Cambridge.

[11] Brand, M. (2006). Fast low-rank modifications of the thin singular value decomposition. Linear algebra and its applications 415(1), 20-30. 
[12] Davis, C. (1957). All convex invariant functions of hermitian matrices. Archiv der Mathematik 8(4), 276-278.

[13] Dinh, T. P. and H. A. Le Thi (2014). Recent advances in dc programming and dca. In Transactions on Computational Intelligence XIII, pp. 1-37. Springer.

[14] Friedman, J., T. Hastie, and R. Tibshirani (2007). Sparse inverse covariance estimation with the graphical lasso. Biostatistics 9, 432-441.

[15] Golub, G. and C. Van Loan (2012). Matrix Computations, Volume 3. JHU Press.

[16] Hiriart-Urruty, J.-B. (1985). Generalized differentiability/duality and optimization for problems dealing with differences of convex functions. In Convexity and duality in optimization, pp. 37-70. Springer.

[17] Jöreskog, K. G. (1967). Some contributions to maximum likelihood factor analysis. Psychometrika 32(4), 443-482.

[18] Larsen, R. Propack-software for large and sparse svd calculations.

[19] Lawley, D. N. (1940). Uthe estimation of factor loadings by the method of maximum likelihood. Proceedings of the Royal Society of Edinburgh 60(01), 64-82.

[20] Lawley, D. N. (1967). Some new results in maximum likelihood factor analysis. Proc.R.Soc. Edinb. 67(01), 256-264.

[21] Lawley, D. N. and A. E. Maxwell (1971). Factor analysis as a statistical method.

[22] Lewis, A. (1996a). Derivatives of spectral functions. Mathematics of Operations Research 21(3), 576-588.

[23] Lewis, A. S. (1996b). Convex analysis on the hermitian matrices. SIAM Journal on Optimization 6, 164-177.

[24] Mardia, K., J. Kent, and J. Bibby (1979). Multivariate Analysis. Academic Press, London.

[25] Nouiehed, M., J.-S. Pang, and M. Razaviyayn (2017). On the pervasiveness of differenceconvexity in optimization and statistics. arXiv preprint arXiv:1704.03535.

[26] Pang, J.-S., M. Razaviyayn, and A. Alvarado (2016). Computing b-stationary points of nonsmooth dc programs. Mathematics of Operations Research 42(1), 95-118.

[27] Pham Dinh, T., H. Ngai, and H. Le Thi (2013). Convergence analysis of dc algorithm for dc programming with subanalytic data. preprint.

[28] Robertson, D. and J. Symons (2007). Maximum likelihood factor analysis with rankdeficient sample covariance matrices. Journal of Multivariate Analysis 98(4), 813-828.

[29] Rockafellar, R. T. and R. J.-B. Wets (2009). Variational analysis, Volume 317. Springer Science \& Business Media.

[30] Rubin, D. B. and D. T. Thayer (1982). Em algorithms for ml factor analysis. Psychometrika $47(1), 69-76$.

[31] Saunderson, J., V. Chandrasekaran, P. Parrilo, and A. Willsky (2012). Diagonal and lowrank matrix decompositions, correlation matrices, and ellipsoid fitting. SIAM Journal on Matrix Analysis and Applications 33(4), 1395-1416.

[32] Shapiro, A. and J. Ten Berge (2002). Statistical inference of minimum rank factor analysis. Psychometrika 67, 79-94.

[33] Spearman, C. (1904). "General Intelligence," Objectively Determined and Measured. American Journal of Psychology 15, 201-293.

[34] Trevor Hastie, R. T. and J. Friedman (2009). The Elements of Statistical Learning, Second Edition: Data Mining, Inference, and Prediction (Springer Series in Statistics) (2 ed.). Springer New York.

[35] Tuy, H. (1995). Dc optimization: theory, methods and algorithms. In Handbook of global optimization, pp. 149-216. Springer.

[36] Vangeepuram, S. and G. B., Lanckriet (2009). On the convergence of the concave-convex procedure. In Advances in Neural Information Processing Systems, (NIPS), Volume 22. MIT Press. 
[37] Yuille, A. and A. Rangarajan (203). The concave-convex procedure (cccp). Neural Computation 15, 915-936. 